POS $\quad$ PROCEEDINGS

\title{
New Higgs physics from the lattice*
}

\section{Zoltan Fodor}

Department of Physics, University of Wuppertal, Gauss Strasse 20, D-42119, Germany

Email: fodorabodri.elte.hu

\section{Kieran Holland}

Department of Physics, University of the Pacific 3601 Pacific Ave, Stockton CA 95211, USA

Email: khollandepacific.edu

\section{Julius Kuti}

Department of Physics 0319, University of California, San Diego 9500 Gilman Drive, La Jolla, CA 92093, USA

E-mail: jkuti@ucsd.edu

\section{Daniel Nogradi}

Department of Physics, University of Wuppertal, Gauss Strasse 20, D-42119, Germany Email: nogradielorentz. leidenuniv.nl

\section{Chris Schroeder}

Department of Physics 0319, University of California, San Diego

9500 Gilman Drive, La Jolla, CA 92093, USA

E-mail: crsephysics.ucsd.edu

We report the first results from our comprehensive lattice tool set to explore non-perturbative aspects of Higgs physics in the Standard Model. We demonstrate in Higgs-Yukawa models that Higgs mass lower bounds and upper bounds can be determined in lattice simulations when triviality requires the necessity of a finite cutoff to maintain non-zero interactions. The vacuum instability problem is investigated and the lattice approach is compared with the traditional renormalization group procedure which sets similar goals to correlate lower and upper Higgs mass bounds with the scale of new physics. A novel feature of our lattice simulations is the use of Ginsparg-Wilson fermions to represent the effects of Top quark loops in Higgs dynamics. The need for chiral lattice fermions is discussed and the approach is extended to full Top-Higgs-QCD dynamics. We also report results from our large $N_{F}$ analysis of Top-Higgs Yukawa models to gain analytic insight and to verify our new lattice tool set which is deployed in the simulations. The role of non-perturbative lattice studies to investigate heavy Higgs particle scenarios is illustrated in extensions of the Standard Model.

The XXV International Symposium on Lattice Field Theory

July 30 - August 42007

Regensburg, Germany

\footnotetext{
*Based on contributions from K. Holland, J. Kuti, D. Nogradi, and C. Schroeder.
} 


\section{Introduction}

The search for the Higgs has become a major issue in particle physics as the LHC is nearing its completion. The Standard Model (SM) cannot be considered complete given that the Higgs is as-yet unobserved and it is not clear how Electroweak symmetry is broken in nature. If the Higgs is seen, its properties could tell us about physics beyond the Standard Model, such as the energy scale of a more fundamental theory. The current lower bound for the Higgs mass from direct searches is $114.4 \mathrm{GeV}$ [1]. The Higgs mass can also be inferred indirectly by fitting the Standard Model to a host of Electroweak precision measurements. The best perturbative fit gives $m_{H}=76_{-24}^{+33} \mathrm{GeV}$, so the data certainly seem to prefer the Higgs to be light [2]. However, the global fitting procedure, which favors a surprisingly low Higgs mass, has its own intrinsic issues, perhaps a hint that deviations from the Standard Model are already present [3]. Larger Higgs masses together with new physics threshold effects at the $\mathrm{TeV}$ scale will require new extended analysis $[4,5]$ where non-perturbative effects may come into play.

Based on the assumption that the Standard Model is only valid up to some energy scale, lower and upper bounds on the Higgs mass were established before without relying on input from Electroweak precision measurements. Bounds on the Higgs mass are valuable for two reasons. Firstly, they cut down the parameter space where one searches for a Standard Model Higgs. Secondly, if the Higgs is found, measuring its mass and knowing the bounds it must obey would indicate the maximum energy scale up to which the Standard Model can work. In phenomenology, the origin of the lower bound is thought to be the vacuum instability the Top quark loop would generate, if the Higgs mass were too light. The upper bound in phenomenological analysis is simply calculated by not allowing the running Higgs coupling $\lambda(t)$ to become strong at the cutoff scale $\Lambda$ which represents new physics before $\lambda(t)$ would run into the fictitious Landau pole. These ideas on lower and upper Higgs mass bounds have been applied to the Standard Model for almost 30 years and have been increasingly refined.

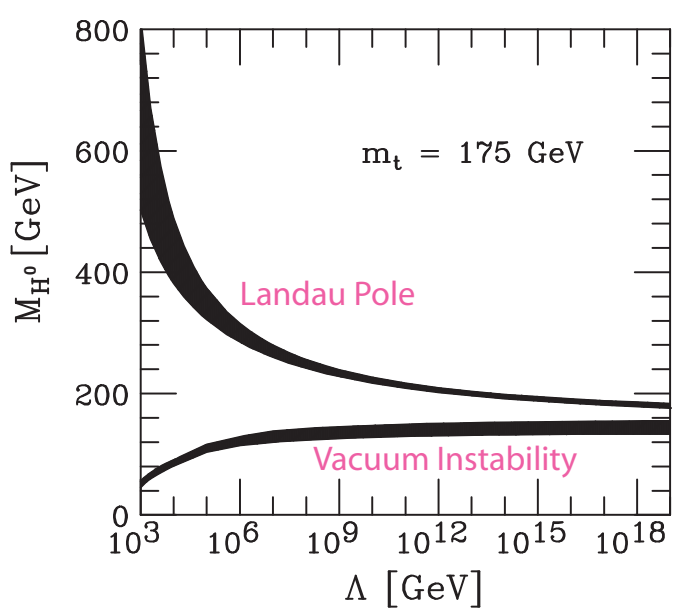

Figure 1: Upper and lower bounds for the Higgs mass as a function of the scale of new physics beyond the Standard Model, from [6].
The bounds given by the state-of-the-art calculations were reviewed in [6] and shown in Figure 1, based on the original work in [7] and [8]. There are several things one can learn from this plot. The Standard Model apparently cannot generate a Higgs boson heavier than $1 \mathrm{TeV}$ without strong Higgs self-interactions and a low threshold for new physics in the $\mathrm{TeV}$ range, a scenario not consistent with the perturbative loop expansion of the Electroweak precision analysis. What non-perturbative modifications on the $\mathrm{TeV}$ scale would support a heavy Higgs particle, consistent with Electroweak precision data, is one of the motivations for our lattice studies $[4,5]$. The lower bound is interesting for today's phenomenology, given the current experimental limits. If the Higgs mass is around $100 \mathrm{GeV}$, this would intersect with the lower bound in Figure 1 somewhere between 
10 and $100 \mathrm{TeV}$, beyond which apparently new physics should enter.

One major goal of our lattice Higgs project is to understand the role of vacuum instability and the Landau pole in an exact non-perturbative setting when the intrinsic cutoff in the Higgs sector is not removable and low in the TeV range. Another goal is to explore the role of nonperturbative Higgs physics from the lattice in extensions of the perturbative SM analysis, including the possibility of a heavy Higgs particle within the Higgs reach of the LHC.

The outline of this paper is as follows. In section 2 we will report results from the large $N_{F}$ analysis of the Top-Higgs Yukawa model of a single real scalar field coupled to $N_{F}$ fermions. The influence of the non-removable intrinsic cutoff (triviality) on the exact renormalization group (RG) flow is exhibited. The vacuum instability problem of the model is discussed on the lattice in section 3 and compared with the traditional renormalization group procedure of the Standard Model (earlier versions of this work on vacuum instability have been discussed in [9] and [10]). In section 4 we present the Wilsonian view on the renormalization group as applied to the vacuum instability and Higgs lower bound problems. The first lattice simulation results on the Higgs mass lower bound, using chiral lattice fermions in Top-Higgs Yukawa models, are reported in section 5.

Using the higher derivative (Lee-Wick) extension of the Higgs sector [11, 12, 13], we will illustrate in section 6 how non-perturbative lattice studies might help to investigate heavy Higgs particle scenarios in the $500-800 \mathrm{GeV}$ Higgs mass range relevant for future LHC physics. Constraints from Electroweak precision data on the heavy Higgs particle are briefly discussed.

\section{Top-Higgs Yukawa model in large $N_{F}$ limit}

For pedagogical purposes, we first consider a Higgs-Yukawa model of a single real scalar field coupled to $N_{F}$ massless fermions. The saddle point approximation in the large $N_{F}$ limit becomes exact and this will allow us to demonstrate that the theory is trivial. We will also calculate the flow of the renormalized couplings as a function of the energy scale to identify problems with the vacuum instability scenario when the intrinsic cutoff is non-removable. Similar behavior is expected at finite $N_{F}$ which requires non-perturbative lattice simulations.

\subsection{Renormalization scheme}

Let us start with the bare Lagrangian of the Higgs-Yukawa theory in Euclidean space-time, which is

$$
\mathscr{L}=\frac{1}{2} m_{0}^{2} \phi_{0}^{2}+\frac{1}{24} \lambda_{0} \phi_{0}^{4}+\frac{1}{2}\left(\partial_{\mu} \phi_{0}\right)^{2}+\bar{\psi}_{0}^{a}\left(\gamma_{\mu} \partial_{\mu}+y_{0} \phi_{0}\right) \psi_{0}^{a}
$$

where $a=1, \ldots, N_{F}$ sums over the degenerate fermion flavors and the subscript 0 denotes bare quantities. We rewrite this as

$$
\begin{aligned}
\mathscr{L}= & \frac{1}{2} m_{0}^{2} Z_{\phi} \phi^{2}+\frac{1}{24} \lambda_{0} Z_{\phi}^{2} \phi^{4}+\frac{1}{2} Z_{\phi}\left(\partial_{\mu} \phi\right)^{2}+Z_{\psi} \bar{\psi}^{a}\left(\gamma_{\mu} \partial_{\mu}+y_{0} \sqrt{Z_{\phi}} \phi\right) \psi^{a} \\
= & \frac{1}{2}\left(m^{2}+\delta m^{2}\right) \phi^{2}+\frac{1}{24}(\lambda+\delta \lambda) \phi^{4}+\frac{1}{2}\left(1+\delta z_{\phi}\right)\left(\partial_{\mu} \phi\right)^{2} \\
& +\left(1+\delta z_{\psi}\right) \bar{\psi}^{a} \gamma_{\mu} \partial_{\mu} \psi^{a}+\bar{\psi}^{a}(y+\delta y) \phi \psi^{a},
\end{aligned}
$$

where we have introduced the wavefunction renormalization factors $Z_{\phi}=1+\delta z_{\phi}, Z_{\psi}=1+\delta z_{\psi}$ and renormalized parameters with their corresponding counterterms. The connections between the 
bare and renormalized parameters are

$$
m_{0}^{2} Z_{\phi}=m^{2}+\delta m^{2}, \quad \lambda_{0} Z_{\phi}^{2}=\lambda+\delta \lambda, \quad Z_{\psi} \sqrt{Z_{\phi}} y_{0}=y+\delta y .
$$

In the limit where $N_{F}$ becomes large, all Feynman diagrams with Higgs loops are suppressed relative to those with fermion loops. Hence, two of the counterterms vanish, $\delta y=0, \delta z_{\psi}=0$, as there are no radiative corrections to the fermion propagator or to the Higgs-fermion coupling. Let us specify the renormalization conditions which determine the remaining counterterms.

In the large $N_{F}$ limit, the renormalized Coleman-Weinberg effective potential [14] is

$$
U_{\text {eff }}=\frac{1}{2} m^{2} \phi^{2}+\frac{1}{24} \lambda \phi^{4}+\frac{1}{2} \delta m^{2} \phi^{2}+\frac{1}{24} \delta \lambda \phi^{4}-2 N_{F} \int_{k} \ln \left[1+y^{2} \phi^{2} / k^{2}\right]
$$

containing the tree-level contributions from the renormalized parameters and their counterterms, and the infinite sum of all diagrams with one fermion loop and an even number of external $\phi$ legs. The factor $N_{F}$ comes from all the possible fermions which can appear in the single loop and we use the notation $\frac{1}{(2 \pi)^{4}} \int d^{4} k \rightarrow \int_{k}$ for loop integrals. The vacuum expectation value $\phi=v$ is where $U_{\text {eff }}$ has an absolute minimum i.e. $U_{\text {eff }}^{\prime}(v)=0$. In the Higgs phase of the theory, $v \neq 0$. At tree-level, this gives the relation

$$
m^{2}+\frac{1}{6} \lambda v^{2}=0
$$

coming from the first two terms in Equation (2.4). Our first renormalization condition is that we want to maintain the tree-level relation in Equation (2.5) exactly, giving

$$
\delta m^{2}+\frac{1}{6} \delta \lambda v^{2}-4 N_{F} y^{2} \int_{k} \frac{1}{k^{2}+y^{2} v^{2}}=0 .
$$

The counterterms exactly cancel all the finite and infinite contributions of the radiative diagrams. The same relation can also be determined by demanding that the tadpole diagram is exactly cancelled by the counterterms.

In the Higgs phase, we define the Higgs fluctuation around the vev as $\phi=\varphi+v$. At tree-level, the mass of the Higgs fluctuation i.e. $U_{\text {eff }}^{\prime \prime}(v)$ is

$$
m_{H}^{2}=m^{2}+\frac{1}{2} \lambda v^{2}=\frac{1}{3} \lambda v^{2} .
$$

In the large $N_{F}$ limit, the inverse propagator of the Higgs fluctuation is

$$
\begin{aligned}
G_{\varphi \varphi}^{-1}\left(p^{2}\right) & =p^{2}+m^{2}+\frac{1}{2} \lambda v^{2}+p^{2} \delta z_{\phi}+\delta m^{2}+\frac{1}{2} \delta \lambda v^{2}-\Sigma\left(p^{2}\right) \\
\Sigma\left(p^{2}\right) & =-4 N_{F} y^{2} \int_{k} \frac{y^{2} v^{2}-k \cdot(k-p)}{\left(k^{2}+y^{2} v^{2}\right)\left((k-p)^{2}+y^{2} v^{2}\right)},
\end{aligned}
$$

where all Higgs-loop diagrams are suppressed relative to the single fermion-loop diagram. We impose the condition that

$$
G_{\varphi \varphi}^{-1}\left(p^{2} \rightarrow 0\right)=p^{2}+m_{H}^{2},
$$

which separates into two renormalization conditions:

$$
\delta m^{2}+\frac{1}{2} \delta \lambda v^{2}-\Sigma\left(p^{2}=0\right)=0
$$


and

$$
\delta z_{\phi}-\left.\frac{d \Sigma\left(p^{2}\right)}{d p^{2}}\right|_{p^{2}=0}=0
$$

The renormalization condition Equation (2.10) maintains the tree-level relation in Equation (2.7) exactly. Again, the counterterms precisely cancel all the finite and infinite radiative contributions. We should point out that the Higgs mass defined as the zero-momentum piece of $G_{\varphi \varphi}^{-1}$ is identical to that defined via the curvature $U_{\mathrm{eff}}^{\prime \prime}(v)$. This is not the same as the true physical mass given by the pole of the propagator, and these masses can be related to one another in perturbation theory.

The renormalization conditions Equations (2.6) and (2.10) can easily be solved. Because we wish to demonstrate triviality in this theory, we use some finite cutoff in the momentum integrals and examine what occurs as this cutoff is removed. We will use a simple hard-momentum cutoff $|k| \leq \Lambda$. Exactly the same conclusions would be reached using instead e.g. Pauli-Villars regularization. The non-zero counterterms after the loop integration are

$$
\begin{aligned}
\delta m^{2} & =\frac{N_{F} y^{2}}{2 \pi^{2}}\left[\frac{1}{2} \Lambda^{2}+\frac{y^{4} v^{4}}{2\left(\Lambda^{2}+y^{2} v^{2}\right)}-\frac{1}{2} y^{2} v^{2}\right], \\
\delta \lambda & =-\frac{3 N_{F} y^{4}}{\pi^{2}}\left[\frac{y^{2} v^{2}}{2\left(\Lambda^{2}+y^{2} v^{2}\right)}-\frac{1}{2}-\frac{1}{2} \ln \left(\frac{y^{2} v^{2}}{\Lambda^{2}+y^{2} v^{2}}\right)\right], \\
\delta z_{\phi} & =-\frac{N_{F} y^{2}}{2 \pi^{2}}\left[\frac{1}{4} \ln \left(\frac{y^{2} v^{2}+\Lambda^{2}}{y^{2} v^{2}}\right)+\frac{-5 \Lambda^{4}-3 \Lambda^{2} y^{2} v^{2}}{12\left(\Lambda^{2}+y^{2} v^{2}\right)^{2}}\right] .
\end{aligned}
$$

As we said earlier, in the large $N_{F}$ limit, the fermion inverse propagator receives no radiative correction,

$$
G_{\psi \psi}^{-1}(p)=p_{\mu} \gamma_{\mu}+y v
$$

so we identify the fermion mass as $m_{t}=y v$ (looking ahead to the Top quark), which we substitute into all of the above equations.

\subsection{Triviality}

Let us first consider the regime $m_{t} / \Lambda \ll 1$, where the cutoff is much larger than the physical scale. In this limit, we get

$$
Z_{\phi}=\left[1+\frac{N_{F} y_{0}^{2}}{8 \pi^{2}}\left(\ln \left[\frac{\Lambda^{2}}{m_{t}^{2}}\right]-\frac{5}{3}\right)\right]^{-1}
$$

For any finite bare Yukawa coupling $y_{0}$, the Higgs wavefunction renormalization factor $Z_{\phi}$ vanishes logarithmically as the cutoff is removed, $m_{t} / \Lambda \rightarrow 0$. This same logarithmic behavior, for any choice of bare couplings, will appear in all of the renormalized couplings, leading to the triviality scenario: a finite cutoff must be kept to maintain non-zero interactions. Explicitly, the renormalized Yukawa coupling is

$$
y^{2}=y_{0}^{2} Z_{\phi}=y_{0}^{2}\left[1+\frac{N_{F} y_{0}^{2}}{8 \pi^{2}}\left(\ln \left[\frac{\Lambda^{2}}{m_{t}^{2}}\right]-\frac{5}{3}\right)\right]^{-1} \rightarrow\left[\frac{N_{F}}{8 \pi^{2}} \ln \frac{\Lambda^{2}}{m_{t}^{2}}\right]^{-1}, \quad \text { as } \frac{m_{t}}{\Lambda} \rightarrow 0 .
$$


For the renormalized Higgs coupling, we have

$$
\begin{aligned}
\lambda & =\lambda_{0} Z_{\phi}^{2}-\delta \lambda=\lambda_{0} Z_{\phi}^{2}+\frac{3 N_{F} y^{4}}{\pi^{2}}\left[\frac{m_{t}^{2}}{2\left(\Lambda^{2}+m_{t}^{2}\right)}-\frac{1}{2}-\frac{1}{2} \ln \left(\frac{m_{t}^{2}}{\Lambda^{2}+m_{t}^{2}}\right)\right] \\
& \rightarrow Z_{\phi}^{2}\left[\lambda_{0}+\frac{3 N_{F} y_{0}^{4}}{\pi^{2}}\left(-\frac{1}{2}-\frac{1}{2} \ln \frac{m_{t}^{2}}{\Lambda^{2}}\right)\right] \rightarrow 12\left[\frac{N_{F}}{8 \pi^{2}} \ln \frac{\Lambda^{2}}{m_{t}^{2}}\right]^{-1}, \quad \text { as } \frac{m_{t}}{\Lambda} \rightarrow 0 .
\end{aligned}
$$

The slow logarithmic vanishing of $y$ and $\lambda$ allows to have a relatively large separation of cutoff and physical scales and still maintain significant interactions. However, the standard renormalization procedure of removing the cutoff completely gives a non-interacting theory. Although completely unphysical, we can also consider the limit $m_{t} / \Lambda \gg 1$, where the cutoff is much below the physical scale. From Equation (2.12), we see this gives $\delta \lambda=0, \delta z_{\phi}=0$, and hence $Z_{\phi} \rightarrow 1$. In this limit, the connection between bare and renormalized parameters is simply $\lambda=\lambda_{0}, y=y_{0}$. This result is not surprising: deep in the cutoff regime, we simply have the bare theory, with no separation into renormalized parameters and their counterterms. This will be relevant when we discuss whether the vacuum can become unstable.

\subsection{Renormalization group flow}

The physical properties of the theory are fixed as soon as one chooses a complete set of bare parameters. As the cutoff is varied, the renormalized couplings flow in order to maintain exactly the renormalization conditions we have imposed. Using the explicit cutoff dependence of $y$ and $\lambda$, we can calculate this Callan-Symanzik flow. In the limit $m_{t} / \Lambda \ll 1$, from Equations (2.15) and (2.16), we have

$$
\begin{aligned}
\Lambda \frac{d y^{2}}{d \Lambda} & =-y_{0}^{2} Z_{\phi}^{2} \frac{N_{F} y_{0}^{2}}{4 \pi^{2}}=-\frac{N_{F} y^{4}}{4 \pi^{2}} \\
\Lambda \frac{d \lambda}{d \Lambda} & =\frac{1}{16 \pi^{2}}\left[-8 N_{F} \lambda y^{2}+48 N_{F} y^{4}\right] .
\end{aligned}
$$

The same $\beta$ functions would be obtained in the large $N_{F}$ limit for the running $y$ and $\lambda$ couplings in scale dependent RG flows using e.g. dimensional regularization, where no cutoff would explicitly appear. (Since increasing $\Lambda$ corresponds to decreasing mass scale $\mu$, the $\beta$ functions in Equation (2.17) have opposite signs). It is important to note that the two RG schemes have very different physical meanings: Equation (2.17) describes the response to changing the cutoff whereas the scale dependent RG flow compensates for the arbitrary choice of the renormalization scale at finite cutoff. When the cutoff is far above the physical scales, the finite cutoff effects are negligible and we expect to reproduce the unique cutoff-independent $\beta$ functions. However, as the cutoff is reduced and $m_{t} / \Lambda$ increases, this cannot continue to hold indefinitely, as the renormalized couplings must eventually flow to the bare ones, as explained above.

Let us demonstrate an explicit example of the Callan-Symanzik RG flow in the presence of a finite cutoff. In the large $N_{F}$ limit, $m_{t}=y v=y_{0} v_{0}$. The bare vev is determined by the minimum of the bare effective potential

$$
U_{\mathrm{eff}, 0}=\frac{1}{2} m_{0}^{2} \phi_{0}^{2}+\frac{1}{24} \lambda_{0} \phi_{0}^{4}-2 N_{F} \int_{k} \ln \left[1+y_{0}^{2} \phi_{0}^{2} / k^{2}\right] .
$$



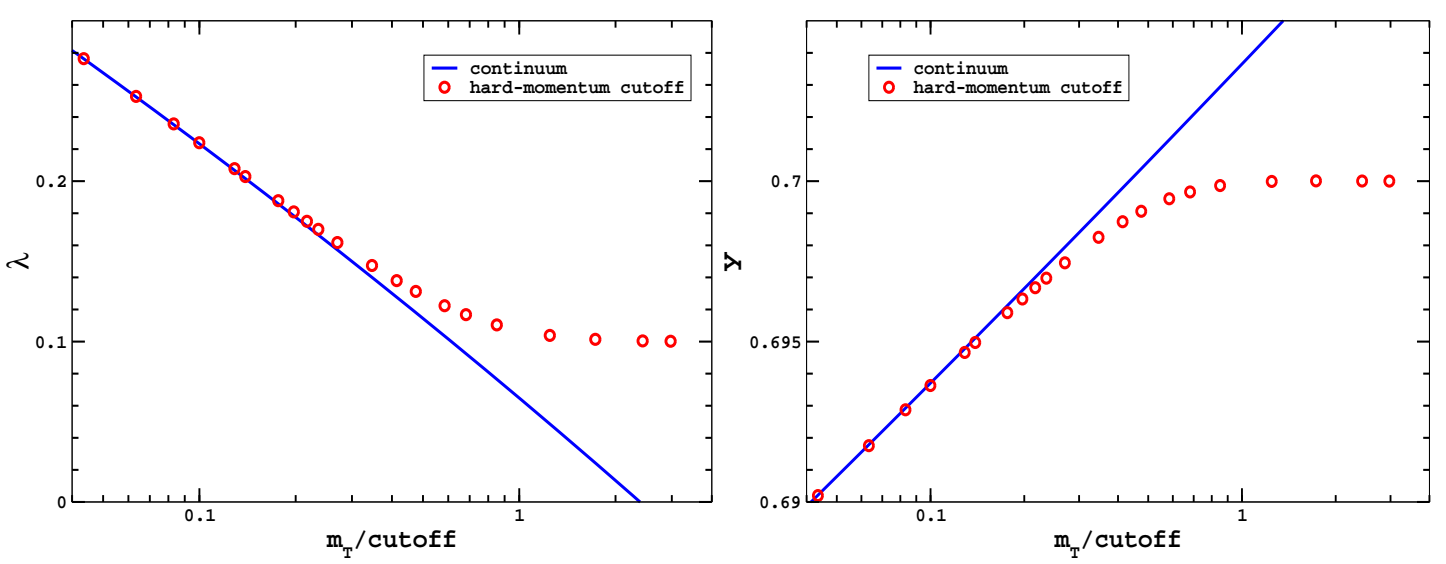

Figure 2: The exact RG flow of the renormalized couplings $\lambda$ and $y$ with the full cutoff dependence. The corresponding bare couplings are $\lambda_{0}=0.1$ and $y_{0}=0.7$. For large cutoff, the exact flow agrees with the continuum RG flow, where the cutoff dependence is omitted. For small cutoff, the exact RG flows to the bare couplings $\lambda_{0}$ and $y_{0}$, but the continuum RG misleadingly predicts that $\lambda$ turns negative.

Using a hard-momentum cutoff, this gives

$$
m_{0}^{2}+\frac{1}{6} \lambda_{0} v_{0}^{2}-\frac{N_{F} y_{0}^{2}}{2 \pi^{2}}\left[\frac{1}{2} \Lambda^{2}+\frac{1}{2} y_{0}^{2} v_{0}^{2} \ln \left(\frac{y_{0}^{2} v_{0}^{2}}{\Lambda^{2}+y_{0}^{2} v_{0}^{2}}\right)\right]=0
$$

We express all dimensionful quantities in units of the cutoff $\Lambda$. We pick some fixed values for $\lambda_{0}$ and $y_{0}$. Varying the value of $m_{0}^{2} / \Lambda^{2}$ changes the solution $v_{0} / \Lambda$ of Equation (2.19) and hence the ratio $m_{t} / \Lambda$. As we said, choosing the values of the bare parameters completely determines everything in the theory. For example, to attain a very small value of $m_{t} / \Lambda$ requires $m_{0}^{2} / \Lambda^{2}$ to be tuned quite precisely. Using Equation (2.19), the critical surface, where $v_{0} / \Lambda=0$, is the transition line

$$
\frac{m_{0}^{2}}{\Lambda^{2}}-\frac{N_{F} y_{0}^{2}}{4 \pi^{2}}=0
$$

Using Equations (2.3) and (2.12), all of the counterterms and renormalized parameters can be expressed in terms of $\lambda_{0}, y_{0}, m_{0}^{2}$ and $v_{0}$. Solving this set of simultaneous equations is a simple numerical exercise. We make an arbitrary choice $\lambda_{0}=0.1, y_{0}=0.7$ which would correspond to the physical Higgs below its lower bound in phenomenological considerations. Varying the value of $m_{0}^{2} / \Lambda^{2}$, we explore numerically the range $10^{-13}<m_{t} / \Lambda<10^{2}$. The results in a limited range are plotted in Figure 2. When the cutoff is high, the exact RG flow is exactly the same as if the cutoff had been completely removed and follows precisely the continuum form of Equation (2.17). However, as the cutoff is reduced, the exact RG flow eventually breaks away from the continuum form and reaches a plateau at the value of the bare coupling.

The continuum RG in the above example predicts that $\lambda$ turns negative at some energy scale as the flow continues. This was used in the past as an indication that the ground state of the theory turns unstable at that scale which would determine the energy scale of new physics necessary to sustain a particular value of the physical Higgs mass (vacuum instability bound). As shown above, the true RG flow with the full cutoff dependence saturates at $\lambda_{0}$ and does not turn negative under the necessary $\lambda_{0}>0$ stability requirement of the model. This makes the phenomenological RG 
method and the apparent vacuum instability quite suspect in the presence of the non-removable finite cutoff which is required by triviality of the renormalized couplings.

The absence of vacuum instability will be demonstrated directly in the next section using the Higgs effective potential. In sections 4 and 5 we will propose a lattice strategy to determine the Higgs mass lower bound in the presence of an intrinsic cutoff without relying on the continuum RG flow. In this new strategy even the $\lambda_{0}>0$ condition might be relaxed by adding new irrelevant operators, like the $\frac{\lambda_{6}}{\Lambda^{2}} \phi^{6}$ term, to keep the stability of the cutoff theory intact.

\section{The effective potential and vacuum instability}

First, we will present here the RG improved one-loop calculation of the effective potential with unstable vacuum when the cutoff is ignored. Next we show the absence of vacuum instability when the cutoff is correctly enforced.

\subsection{Continuum 1-loop effective potential}

For the Higgs-Yukawa model with $N_{F}$ fermions of Section 2, the 1-loop renormalized effective potential is

$$
\begin{aligned}
U_{\text {eff }} & =\frac{1}{2} m^{2} \phi^{2}+\frac{1}{24} \lambda \phi^{4}+\frac{1}{2} \delta m^{2} \phi^{2}+\frac{1}{24} \delta \lambda \phi^{4}-2 N_{F} \int_{k} \ln \left[1+y^{2} \phi^{2} / k^{2}\right] \\
& +\frac{1}{2} \int_{k}\left(\ln \left[k^{2}+V^{\prime \prime}(\phi)\right]-\ln \left[k^{2}+V^{\prime \prime}(0)\right]\right), \\
V & =\frac{1}{2} m^{2} \phi^{2}+\frac{1}{24} \lambda \phi^{4},
\end{aligned}
$$

where the Higgs-loop contributions are also included now. For consistency, we impose exactly the same renormalization conditions Equations (2.10) and (2.11) used in Section 2, including all the Higgs-loop radiative corrections. Because $\delta y$ and $\delta z_{\psi}$ are non-zero (we no longer impose the large $N_{F}$ limit), we specify the two additional renormalization conditions. The fermion inverse propagator is

$$
\begin{array}{r}
G_{\psi \psi}^{-1}(p)=p_{\mu} \gamma_{\mu}+y v+\delta z_{\psi} p_{\mu} \gamma_{\mu}+\delta y v-\Sigma_{F}(p), \\
\Sigma_{F}(p)=y^{2} \int_{k} \frac{-k_{\mu} \gamma_{\mu}+y v}{\left(k^{2}+y^{2} v^{2}\right)\left((k-p)^{2}+m_{H}^{2}\right)},
\end{array}
$$

the radiative correction coming from a single Higgs-loop diagram, and we require that

$$
G_{\psi \psi}^{-1}(p \rightarrow 0)=p_{\mu} \gamma_{\mu}+y \nu
$$

This gives two renormalization conditions,

$$
\begin{aligned}
& \delta y v-\Sigma_{F}(p \rightarrow 0)=0, \\
& \delta z_{\psi}-\left.\frac{d \Sigma_{F}}{d\left(p_{\mu} \gamma_{\mu}\right)}\right|_{p \rightarrow 0} .
\end{aligned}
$$

Again, the counterterms completely remove all the finite and infinite parts of the radiative corrections. We regulate the momentum integrals using e.g. a hard-momentum cutoff. The counterterms 
and the renormalized effective potential are calculated exactly using a finite cutoff. We then take the naive limit $\phi / \Lambda \rightarrow 0$ to remove all cutoff dependence. This ignores the fact that a finite and possibly low cutoff is required to maintain $\lambda, y \neq 0$ (a crucial point why the instability does not occur in the presence of finite cutoff).

The continuum form of the 1-loop renormalized effective potential is given by

$$
\begin{aligned}
U_{\text {eff }}= & \frac{1}{2} m^{2} \phi^{2}+\frac{1}{24} \lambda \phi^{4}-\frac{N_{F} y^{4}}{16 \pi^{2}}\left[-\frac{3}{2} \phi^{4}+2 v^{2} \phi^{2}+\phi^{4} \ln \frac{\phi^{2}}{v^{2}}\right] \\
+ & \frac{1}{16 \pi^{2}}\left[\frac{1}{16}\left(\lambda^{2} \phi^{4}-2 \lambda \phi^{2} m_{H}^{2}\right) \ln \frac{m^{2}+\lambda \phi^{2} / 2}{m_{H}^{2}}\right. \\
& \left.+\frac{1}{16} m_{H}^{4} \ln \frac{m^{2}+\lambda \phi^{2} / 2}{m^{2}}-\frac{3}{32} \lambda^{2} \phi^{4}+\frac{7}{16} \lambda \phi^{2} m_{H}^{2}\right],
\end{aligned}
$$

where $m_{H}^{2}=\lambda v^{2} / 3$. Due to our choice of renormalization conditions, the tree-level vev $v=$ $\sqrt{3 m_{H}^{2} / \lambda}$ is not shifted: one can check explicitly that $U_{\text {eff }}$ in Equation (3.5) has its minimum at $\phi=v$. The large $N_{F}$ limit can be recovered by omitting the Higgs-loop terms.

\subsection{RG improved effective potential and vacuum instability}

The stability of the ground state is determined by the behavior of $U_{\text {eff }}$ for large $\phi$. We see from Equation (3.5) that the dominant terms in this regime are of the form $\lambda^{2} \phi^{4} \ln \left(\phi^{2} / v^{2}\right)$ and $-N_{F} y^{4} \phi^{4} \ln \left(\phi^{2} / v^{2}\right)$. The negative fermion term brings up the possibility that the vev $v$ is unstable. Hence stability is determined by the relative values of $\lambda^{2}$ and $y^{4}$, which are related to $m_{H}$ and $m_{t}$. If the fermionic term dominates at large $\phi$, the minimum at $v$ is only a local one and will decay. If we believe that the vacuum is absolutely stable, then new degrees of freedom must enter at the scale where $U_{\text {eff }}(\phi)$ first becomes unstable. For given values of $m_{H}$ and $m_{t}$, this predicts the emergence of new physics. Turning this around, let us fix $m_{t}$ and ask that no new stabilizing degrees of freedom are needed for $\phi \leq E$. Then we obtain a lower bound $m_{H}(E)$ : if the Higgs is lighter than this, $U_{\text {eff }}$ is already unstable for $\phi$ below $E$ because the fermion term dominates even earlier.

Improved vacuum instability can be shown via the running renormalized couplings in RG setting. We can define a set of renormalization conditions in the continuum, for example in the $\overline{M S}$ scheme, where the couplings flow with the renormalization scale $\mu$. The 1-loop RG equations for the Higgs-Yukawa model are

$$
\begin{aligned}
& \mu \frac{d y}{d \mu}=\frac{1}{8 \pi^{2}}\left(3+2 N_{F}\right) y^{4}, \\
& \mu \frac{d \lambda}{d \mu}=\frac{1}{16 \pi^{2}}\left(3 \lambda^{2}+8 N_{F} \lambda y^{2}-48 N_{F} y^{4}\right) .
\end{aligned}
$$

We can set the initial conditions $\lambda(\mu=v)=3 m_{H}^{2} / v^{2}$ and $y(\mu=v)=m_{t} / v$. If $m_{t}$ is sufficiently heavy relative to $m_{H}$, the Yukawa coupling dominates the RG flow and $d \lambda / d \mu<0$. The renormalized Higgs coupling eventually becomes negative at some $\mu=E$. If the instability occurs at very large $\phi / v$, large logarithmic terms $\ln (\phi / v)$ in $U_{\text {eff }}$ might spoil the perturbative expansion. This can be reduced using renormalization group improvement to resum the leading large logarithms. The dominant terms of $U_{\text {eff }}$ at large $\phi$ then become $\lambda(\mu) \phi^{4}(\mu)$. Hence $\lambda(E)=0$ indicates that the ground state is just about to become unstable. 


\subsection{The constraint effective potential on the lattice}

We can calculate the exact effective potential non-perturbatively, using lattice simulations. This was first shown in the pure Higgs theory by Kuti and Shen [15]. There is some finite lattice spacing $a$ on the lattice which restricts the momenta $\left|p_{\mu}\right| \leq \pi / a$ replacing the sharp momentum cutoff used in section 2. For a Higgs-Yukawa theory with $N_{F}$ fermions, the Euclidean lattice partition function is

$$
\begin{aligned}
& Z=\prod_{x} \int d \phi_{0}(x)\left[\operatorname{Det}\left(D\left[\phi_{0}\right]\right)\right]^{N_{F}} \exp \left(-S\left[\phi_{0}\right]\right),=\prod_{x} \int d \phi_{0}(x) \exp \left(-S_{\mathrm{eff}}\left[\phi_{0}\right]\right) \\
& S=\sum_{x} \frac{1}{2} m_{0}^{2} \phi_{0}^{2}(x)+\frac{1}{24} \lambda_{0} \phi_{0}^{4}(x)+\frac{1}{2}\left(\partial_{\mu} \phi_{0}(x)\right)^{2}, \\
& \left(D\left[\phi_{0}\right]\right)_{x y}=\gamma_{\mu} \partial_{\mu, x y}+y_{0} \phi_{0}(x) \delta_{x y},
\end{aligned}
$$

where the partial derivatives are replaced by finite lattice differences. If the integrand is positivedefinite, it can be interpreted as a probability density and importance sampling (i.e. Monte Carlo integration) can be used to calculate expectation values, e.g. $\left\langle\phi_{0}\right\rangle$, non-perturbatively with the exact distribution $[\operatorname{Det}(D)]^{N_{F}} \exp (-S)$. All dimensionful quantities are calculated in units of the lattice spacing $a$. There is a phase diagram in the bare-coupling space $m_{0}^{2}, \lambda_{0}, y_{0}$. The Higgs phase and the symmetric phase are separated by a second order transition, where the vev, $v a$, and the masses $m_{H} a$ and $m_{t} a$, vanish. Since the vev and masses are non-zero in physical units, the transition corresponds to the continuum limit $a \rightarrow 0$. To make the cutoff $\Lambda=\pi / a$ large, the bare couplings must be tuned to be close to the transition line. If we calculate via simulations that e.g. $a v=\langle a \phi\rangle \approx 0.05$ for some choice of bare couplings, we can use $v=246 \mathrm{GeV}$ to convert this into a cutoff $\Lambda \approx 15 \mathrm{TeV}$, as well as determine $m_{H}$ and $m_{t}$ in physical units.

In a finite space-time volume $\Omega$, we will use the constraint effective potential $[15,16]$. For a pure scalar field theory, this is

$$
\exp \left(-\Omega U_{\Omega}(\Phi)\right)=\prod_{x} \int d \phi(x) \delta\left(\Phi-\frac{1}{\Omega} \sum_{x} \phi(x)\right) \exp (-S[\phi]) .
$$

The delta function enforces the constraint that the scalar field $\phi$ fluctuates around a fixed average $\Phi$. The constraint effective potential $U_{\Omega}(\Phi)$ has a very physical interpretation. If the constraint is not imposed, the probability that the system generates a configuration where the average field takes the value $\Phi$ is

$$
P(\Phi)=\frac{1}{Z} \exp \left(-\Omega U_{\Omega}(\Phi)\right), \quad Z=\int d \Phi^{\prime} \exp \left(-\Omega U_{\Omega}\left(\Phi^{\prime}\right)\right) .
$$

This is in very close analogy to the probability distribution for the magnetization in a spin system. The scalar expectation value $v=\langle\phi\rangle$ is the value of $\Phi$ for which $U_{\Omega}$ has an absolute minimum. In a finite volume, the constraint effective potential is non-convex and can have multiple local minima [17]. The standard effective potential $U_{\text {eff }}(\Phi)$ is always convex, even in a finite volume, as the Maxwell construction connects the various minima. The two effective potentials are identical in the infinite-volume limit, $\lim _{\Omega \rightarrow \infty} U_{\Omega}(\Phi)=U_{\text {eff }}(\Phi)$, and the constraint effective potential recovers the convexity property. In a finite volume, it is more useful to work with the constraint effective potential, where multiple minima can be observed and the transition between the Higgs and symmetric phases is clear. It is also more natural, as the probability distribution $P(\Phi)$ can be directly observed in lattice simulations. For the rest of this paper, we drop the subscript $\Omega$. 


\subsection{Hybrid Monte Carlo algorithm and the effective potential}

One way to extract the effective potential from lattice simulations is to generate the ensemble of configurations, calculate the average scalar field $\Phi$ for each configuration and hence the probability distribution $P(\Phi)$. The effective potential is extracted by numerically fitting $U_{\text {eff }}(\Phi)$ to $P(\Phi)$ using Equation (3.9). This gives the effective potential for all $\Phi$ from one simulation, but with limited accuracy. An alternative method is calculate the derivative of the effective potential. For the Higgs-Yukawa model with $N_{F}$ degenerate fermions, the derivative is

$$
\frac{d U_{\text {eff }}}{d \Phi}=m^{2} \Phi+\frac{1}{6} \lambda\left\langle\phi^{3}\right\rangle_{\Phi}-N_{F} y\langle\bar{\psi} \psi\rangle_{\Phi}, \quad\langle\bar{\psi} \psi\rangle_{\Phi}=\left\langle\operatorname{Tr}\left(D[\phi]^{-1}\right)\right\rangle_{\Phi} .
$$

The expectation values $\langle\ldots\rangle_{\Phi}$ mean that, in the lattice simulations, the scalar field fluctuates around some fixed average value $\Phi$. This method determines the effective potential with greater accuracy than fitting the distribution $P(\Phi)$, but the drawback is that a separate lattice simulation has to be run for every value of $\Phi$. This is the method we use in our investigation of the vacuum instability.

In this section we use staggered fermions $[18,19]$, one flavor of which corresponds to four fermion flavors in the continuum. With one staggered fermion, the determinant $\operatorname{Det}(D)$ is real but can be negative due to $\phi$ fluctuations. Then the partition function integrand is not positivedefinite and Monte Carlo integration cannot be applied. To overcome this problem, we simulate two staggered fermions, corresponding to eight continuum flavors, as $[\operatorname{Det}(D)]^{2}$ guarantees a positivedefinite density.We used staggered fermions only in the very early phase of our simulations. The complicated taste structure of staggered fermions with the related rooting issues and the lack of full chiral symmetry motivated the switch to chiral overlap fermions which are used now exclusively in our Higgs project. Staggered results for the effective potential, which are used here mainly for simplicity and pedagogy, have been replaced by simulations with chiral overlap fermions.

Configurations are generated using the Hybrid Monte Carlo algorithm [20], where a fictitious time $t$ and momenta $\pi(x, t)$ are introduced. New configurations are generated from the equations of motion

$$
\begin{aligned}
& \dot{\phi}(x, t)=\pi(x, t), \\
& \dot{\pi}(x, t)=-\left[\frac{\partial S_{\mathrm{eff}}}{\partial \phi(x, t)}-\frac{1}{\Omega} \sum_{y} \frac{\partial S_{\mathrm{eff}}}{\partial \phi(y, t)}\right],
\end{aligned}
$$

where the effective action $S_{\text {eff }}$ is given in Equation (3.7). The second term in $\dot{\pi}(x, t)$ is included to enforce the constraints

$$
\frac{1}{\Omega} \sum_{y} \phi(y, t)=\Phi, \quad \sum_{y} \pi(y, t)=0 .
$$

We work with fixed lattice volumes of size $8^{3} \times 16$. The scalar field has periodic boundary conditions, the fermionic field is periodic in the short directions and antiperiodic in the long direction. We use the standard leapfrog method to solve the equations of motion, where the step-size $\Delta t$ is adjusted to achieve acceptance rates well above $90 \%$, and each trajectory length satisfies $N_{t} \Delta t \geq 1$. For each simulation, we generate at least $10^{4}$ configurations and check that correlations between the configurations are small.

The basic quantities of the theory are the bare fields and couplings. A particular choice of bare couplings puts us somewhere in the phase diagram and all physical quantities are now fixed. 
A separate constrained simulation is run for each value of $\Phi_{0}$ to calculate the effective potential derivative. The expectation values we measure on the lattice are bare ones, so the simulations give the bare equivalent of Equation (3.10), namely

$$
\frac{d U_{\text {eff }}}{d \Phi_{0}}=m_{0}^{2} \Phi_{0}+\frac{1}{6} \lambda_{0}\left\langle\phi_{0}^{3}\right\rangle_{\Phi_{0}}-N_{F} y_{0}\left\langle\bar{\psi}_{0} \psi_{0}\right\rangle_{\Phi_{0}}, \quad\left\langle\bar{\psi}_{0} \psi_{0}\right\rangle_{\Phi_{0}}=\left\langle\operatorname{Tr}\left(D\left[\phi_{0}\right]^{-1}\right)\right\rangle_{\Phi_{0}}
$$

which is converted using the relationship between the bare and renormalized fields,

$$
\Phi=\frac{\Phi_{0}}{\sqrt{Z}_{\phi}}, \quad \frac{d U_{\text {eff }}}{d \Phi}=\sqrt{Z}_{\phi} \frac{d U_{\text {eff }}}{d \Phi_{0}} .
$$

We measure the wave function renormalization factor $Z_{\phi}$ in separate unconstrained simulations.

We want to follow the behavior of $U_{\text {eff }}$ as we approach the continuum limit, the critical surface in the bare-coupling space. We make an arbitrary choice $y_{0}=0.5$ and $\lambda_{0}=0.1$. The distance from the continuum limit is determined by the remaining bare coupling $m_{0}^{2}$. We obtained results for three choices $m_{0}^{2}=0.1,0.25$ and 0.29. Typical non-perturbative measurements of the derivative $d U_{\text {eff }} / d \Phi$ are shown in Figure 3. All dimensionful quantities are in lattice units, e.g. $a \cdot \Phi$. What do we expect to see? In the Higgs phase of the theory, $U_{\text {eff }}$ should have a local maximum at the origin and a local minimum for some non-zero $a \cdot \Phi$. If the vacuum is stable, the local minimum is in fact an absolute one. Let us first look at the results for $m_{0}^{2}=0.1$, shown in Figure 3. The simulations show that $d U_{\text {eff }} / d \Phi$ vanishes at the origin and at $a \cdot \Phi \approx 2.0$; these are the extrema. The derivative is negative between these points, so the origin is indeed a local maximum. For $a \cdot \Phi>2$, the derivative is always positive and the local minimum appears to be an absolute one. If the vacuum is unstable, $d U_{\text {eff }} / d \Phi$ should turn negative at large $a \cdot \Phi$, for which the simulations show no evidence. In these units, the lattice cutoff is $\Lambda=\pi / a$ and the ratio of cutoff to scalar expectation value is $\Lambda / v \approx 1.5$. This is far from the continuum limit.
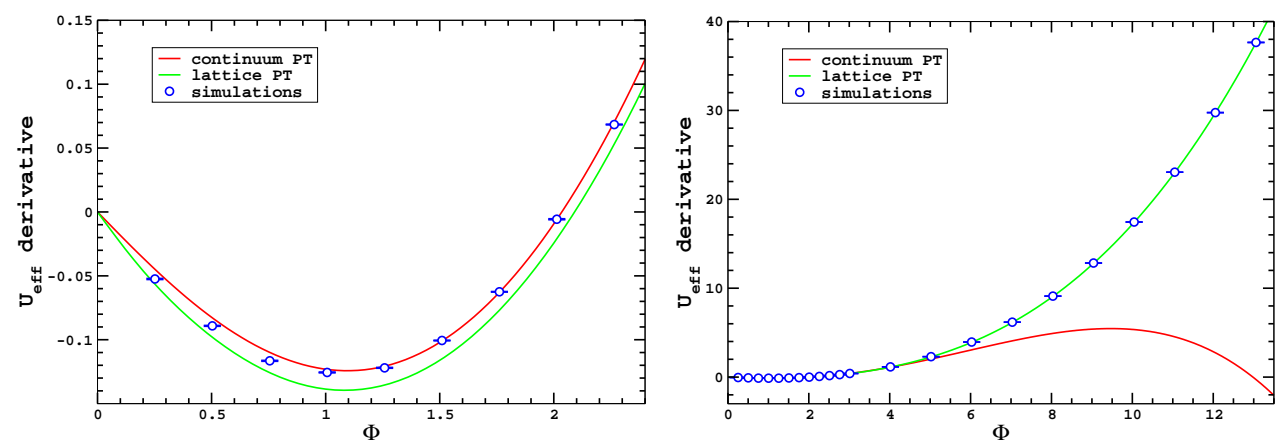

Figure 3: The derivative of the effective potential $d U_{\text {eff }} / d \Phi$ for the bare couplings $y_{0}=0.5, \lambda_{0}=0.1, m_{0}^{2}=$ 0.1 , for which the vev is $a v=2.035(1)$. The left side plot is a close-up of the behavior near the origin. The circles are the results of the simulations and the curves are given by continuum and lattice renormalized perturbation theory.

We vary the bare mass to get closer to the critical surface and the continuum limit for bare masses $m_{0}^{2}=0.25$ and 0.29 respectively. The simulations show the same qualitative behavior for $U_{\text {eff }}$ : the origin is a local maximum, there is an absolute minimum for some non-zero $a \Phi$ and no 


\begin{tabular}{ccccccc}
\hline \hline$y_{0}$ & $\lambda_{0}$ & $m_{0}^{2}$ & $Z_{\phi}$ & $a v=\langle a \phi\rangle$ & $a m_{H}$ & $a m_{t}$ \\
\hline \hline 0.5 & 0.1 & 0.1 & $0.987(1)$ & $2.035(1)$ & $0.521(5)$ & $0.9977(5)$ \\
& & 0.25 & $0.9705(8)$ & $0.811(1)$ & $0.297(4)$ & $0.3906(7)$ \\
& & 0.29 & $0.9676(7)$ & $0.4685(6)$ & $0.248(3)$ & $0.2230(3)$ \\
\hline
\end{tabular}

Table 1: The wave function renormalization factor, the renormalized scalar expectation value and the Higgs and Top masses, obtained from unconstrained lattice simulations. The bare couplings are those used for the

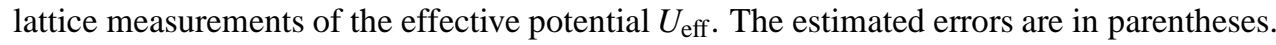

sign of an instability in the potential. The minimum occurs at $a \Phi \approx 0.81$ and 0.47 respectively, for which $\Lambda / v \approx 3.9$ and 6.7 , pushing towards the continuum limit.

The first check of these calculations is to run separate unconstrained simulations with the same bare couplings, where $\sum_{x} \phi(x)$ is allowed to fluctuate freely, and to measure independently $v=\langle\phi\rangle$. This expectation value should be identical to the value of $\Phi$ where $U_{\text {eff }}$ has an absolute minimum, as determined by the constrained simulations. In the unconstrained simulations, the second term for $\dot{\pi}(x, t)$ in Equation (3.11) is omitted. The results of the unconstrained simulations are given in Table 1. There is indeed perfect agreement between the measurements of $\langle a \phi\rangle$ and the location of the $U_{\text {eff }}$ minimum obtained from the constrained simulations. The continuum perturbation theory calculation of $U_{\text {eff }}$ is also shown in Figure 3. We only display the large $N_{F}$ result: not surprisingly, for $N_{F}=8$, the Higgs-loop contributions are negligible and can be omitted. We see excellent agreement with the non-perturbative simulations for $\Phi \lesssim v$, as shown in the left side plot. However, the behavior as $\Phi$ increases is completely different, as shown in the right side plot. Continuum perturbation theory breaks away from the simulation results and predicts that the vacuum becomes unstable, with $d U_{\text {eff }} / d \Phi$ turning negative. The exact non-perturbative calculation shows no indication of this.

What can we conclude from the comparison? Continuum perturbation theory works well for $\Phi$ less than and even close to the lattice cutoff $\Lambda=\pi / a$, as shown by the very good agreement with the exact lattice calculations. This is the most that one could have expected. The instability is predicted at $\Phi$ well above the cutoff, which is completely unphysical and where one cannot expect the continuum calculation to apply. The exact effective potential, with the full cutoff dependence, is absolutely stable. The standard interpretation of the instability in the continuum $U_{\text {eff }}$ would be to say new physics appears at this energy scale to stabilize the ground state. But the actual cutoff of the field theory is far below this scale, especially as we get closer to the continuum limit. The instability only appears when the finite cutoff effects are ignored - there is no need for new physics. One can ask, is it possible to arrange both the standard ground state and the instability to occur well below the regulator cutoff? If so, the instability would be a genuine low-energy prediction. The answer is no in the Top-Higgs Yukawa model, if only the standard terms are included in the lattice Lagrangian. In this case the only freedom one has is the choice of the bare couplings, and nowhere in the coupling-space is a genuine instability seen. If higher dimensional operators are included, the $\lambda_{0}>0$ condition perhaps could be relaxed by adding new irrelevant operators, like the $\frac{\lambda_{6}}{\Lambda^{2}} \phi^{6}$ term, to keep the stability of the cutoff theory intact. This scenario requires further investigation.

It can be shown in renormalized lattice perturbation theory that the breakdown of continuum perturbation theory is due solely to the finite cutoff. A finite cutoff is used in the lattice momentum 
integrals for the radiative corrections and the counterterms of $U_{\text {eff }}$, but otherwise the procedure is the same as in the continuum. In Figure 3 we see excellent agreement between simulations, and lattice and continuum renormalized perturbation theory for $\Phi / v \lesssim 1$. As $\Phi$ increases, lattice perturbation theory exactly tracks the non-perturbative result, showing a perfectly stable ground state. The continuum calculation breaks down, not because of large couplings, but because of the neglected finite cutoff.

\section{Wilsonian renormalization group and vacuum instability}

Most of the original work on the consistency of quantum field theory considered only idealized theories, supposedly fundamental to describe physics at arbitrarily high energies. Although in the previous section on vacuum instability and the related Higgs lower bound problem we found a non-removable intrinsic cutoff, the analysis was based on the traditional renormalization procedure. The Wilsonian viewpoint of the renormalization group provides a broader and more complete perspective on the discussion.

\subsection{Wilson's running Lagrangian}

In the 1970s Wilson developed a new, intuitive way of looking at the renormalization of quantum field theories based on the flow of effective Lagrangians as generated by renormalization group transformations [21]. This is based on the realization that physics as we know it seems to be described by effective quantum field theories, which are useful only up to the energy scale $\Lambda_{0}$ where new and yet unknown physics is reached. Some smooth intrinsic regularization is introduced (inherited from new UV physics) at $\Lambda_{0}$ which in Euclidean space restricts the length $p^{2}$ of all fourmomenta. Physics below the cutoff scale $\Lambda_{0}$ is described by a very general 'bare' Lagrangian $\mathscr{L}\left(\Lambda_{0}\right)$ with an infinite series of local terms, constrained only by symmetries. For any choice of the coupling constants in the local terms of the bare Lagrangian, the Euclidean path integral of the partition function has to be finite and well defined. The most fundamental constraint on the bare Lagrangian is the existence and stability of the functional integral which defines the Euclidean partition function. If the viewpoint of 'naturalness' is adopted, all the coupling constants of the higher dimensional operators are chosen to be of order one in units of $\Lambda_{0}$. Using Wilson's exact renormalization group we can consider smoothly lowering the regularization scale to some value $\Lambda_{R}$ say, of order the energy scale $E$ far below $\Lambda_{0}$. To keep physics unchanged, the coupling constants must change with the regularization scale. Hence we have a running, or effective Lagrangian $\mathscr{L}(\Lambda)$, which flows with $\Lambda$ and remains stable at every stage of the procedure in the sense of a convergent Euclidean path integral. Since we can use the Lagrangian $\mathscr{L}\left(\Lambda_{R}\right)$ to calculate low energy physics at the scale $E$, it is not the coupling constants at $\Lambda_{0}$ that are important, but those at the scale $\Lambda_{R}$. The bare couplings have to be close to a critical surface if $m_{p h} / \Lambda_{0} \ll 1$ for the low energy physical masses $m_{p h}$ of the theory.

An effective field theory is renormalizable if we can calculate all the S-matrix elements for processes with energy scale $E$, up to small errors which vanish as powers of $E / \Lambda_{0}$, once we have determined a finite number of coupling constants at some renormalization scale $\Lambda_{R} \sim E$. These coupling constants are called relevant; all others are irrelevant. Whatever values we choose for $\Lambda_{0}$ (as long as it is large enough) and the irrelevant bare couplings $\eta\left(\Lambda_{0}\right)$ (as long as they are natural 
enough), for a particular choice of the relevant operator set $\lambda\left(\Lambda_{R}\right)$, the irrelevant operator set $\eta\left(\Lambda_{R}\right)$ will be of the order of some power of $\left(\Lambda_{R} / \Lambda_{0}\right)$. In other words, for any point on the submanifold of relevant couplings at $\Lambda_{R}$ there is a flow towards it from a wide variety of initial Lagrangians at $\Lambda_{0}$, all of these being equivalent as far as the values of $\mathrm{S}$-matrix elements for processes with energies of order $E \sim \Lambda_{R}$ are concerned. This more general aspect of renormalizability is the concept of universality. An effective quantum theory thus gives us a much more general notion of renormalizability than we had in conventional quantum field theory: the regularization need no longer be removed, and the irrelevant bare couplings need not be zero.

It is useful now to adopt the Wilsonian view on the running effective Lagrangian to the TopHiggs Yukawa model we investigated in the previous section.

\subsection{Top-Higgs Yukawa model, vacuum instability, and running Lagrangian}

Adapting the notion of the the running Wilson Lagrangian for the Top-Higgs Yukawa model, there are only two marginally irrelevant couplings, $\lambda(t)$ and $y(t)$, in addition to the relevant Higgs mass operator. It is important to note that the couplings for increasing $t=\log \left(\Lambda_{0} / \Lambda\right)$ flow from bare $\lambda_{0}$ and $y_{0}$ toward their low energy renormalized values as a function of the energy scale. For example, in the large $N_{F}$ limit and for large $t$ values, neglecting the irrelevant couplings, the flows are expected to look approximately the same as described by Equation (2.17). The Yukawa coupling $y(t)$ will monotonically decrease from its bare value $y_{0}$ towards zero, at the logarithmic rate of Equation (2.15) for large $t$. The Higgs coupling will start from its bare value $\lambda(0)=\lambda_{0}$ and either it will monotonically decrease, or after some initial rising it will turn around and continue to decrease monotonically towards zero, at the logarithmic rate of Equation (2.16) for large $t$. In the Wilsonian picture, all RG trajectories flow from the general coupling constant space of cutoff Lagrangians $\mathscr{L}\left(\Lambda_{0}\right)_{\text {Top-Higgs }}$ towards the trajectory specified by (2.15) and (2.16) with small but calculable corrections from irrelevant operators in the large $t$ limit.

In the Wilsonian view of the running Lagrangian, the cutoff dependent Higgs mass lower bound can be determined in the space of the bare cutoff Lagrangians $\mathscr{L}\left(\Lambda_{0}\right)_{\text {Top-Higgs }}$ from the smallest allowed value of $\lambda\left(\Lambda_{R}\right)$ for a fixed $\Lambda_{0} / \Lambda_{R} \ll 1$ ratio where a natural choice for $\Lambda_{R}$ is the weak boson mass $m_{Z}$, or the vacuum expectation value $v$. This calculation is, of course, very hard to implement operationally with a large number of bare couplings. The important stability condition is the only constraint (with, or without naturalness) on the space of cutoff Lagrangians. For example, the choice of $\lambda_{0}<0$ a priori should not be excluded at the cutoff scale $\Lambda_{0}$, but it requires the presence of some positive higher dimensional operator, like $\lambda_{0}^{(6)} / \Lambda^{2} \cdot \phi^{6}$, with $\lambda_{0}^{(6)}>0$, to provide stability. Whether the Higgs mass lower bound will be necessarily associated with the limit $\lambda_{0} \rightarrow 0$, or the $\lambda_{0}<0$ region also needs to be explored remains an unresolved and interesting question.

In phenomenological applications an attempt is always made to simplify Wilson's framework of dealing with the full space of running Lagrangians. Invoking the $\Lambda_{0} / \Lambda_{R} \rightarrow 0$ limit, only the running of the relevant and marginally irrelevant couplings is calculated and the effects of irrelevant operators are ignored. In addition, in the application of RG equations to the vacuum instability problem, the simplified equations on $\lambda(t)$ and $y(t)$ are running backward from the $m_{Z}$ scale towards the cutoff $\Lambda_{0}$. This interchange of the natural Wilsonian UV $\rightarrow$ IR flow with the IR $\rightarrow$ UV integration of relevant couplings only is a nontrivial proposition because the Wilsonian RG flow is not 
known to be reversible, and to set all the irrelevant couplings to zero at the scale $\Lambda_{R}=m_{Z}$ would require an unknown extension of the space of cutoff Lagrangians $\mathscr{L}\left(\Lambda_{0}\right)_{\text {Top-Higgs }}$, if it exists at all.

In most of the phenomenological RG applications this is not a problem. We believe, however, that the RG treatment of the vacuum instability problem requires special care. What corresponds to the unstable $U_{\text {eff }}$ in Figure 3 is the running $\lambda(t)$ which at some scale $t_{0}$, far below the cutoff scale, turns negative as the RG is running backward, from $t=\log \Lambda_{0} / m_{Z}$ towards the cutoff scale $t=1$. It is a signal that higher dimensional operators must play a role to provide a continued stability to the theory on all scales. It is unlikely that a positive $\lambda_{0}$ on the cutoff scale can support this picture, forcing the running $\lambda(t)$ to turn positive again and produce an effective potential which will turn back positive again after a second minimum which might be lower than the original one where the spontaneously broken theory was built (decay of the false vacuum). It is more likely that this scenario, if it exists at all, will require the $\lambda_{0}<0$ extension of the space of bare Lagrangians. This is an extension which remains largely unexplored and we are just beginning to investigate it.

\subsection{Phenomenology from 2-loop continuum RG}

Vacuum instability was first raised in [22] and it has since been increasingly refined in application to the Standard Model [23, 24, 25, 26, 27, 28, 29, 30, 31, 32, 33, 34, 8, 35, 36]. The state-of-the-art calculation determines the effective potential to one-loop order, with RG improvement applied up to two-loop order to the running couplings.

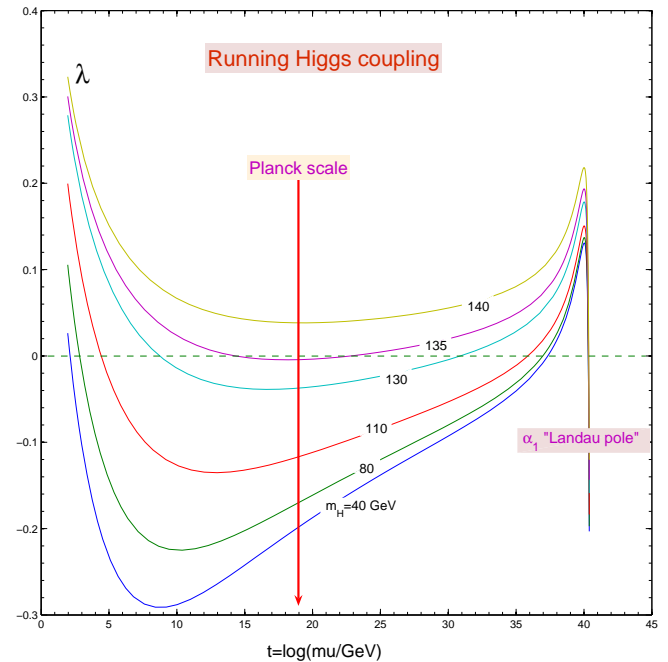

Figure 4: The running Higgs coupling is plotted for different choices of the Higgs mass from our numerical solution of the five coupled 2-loop RG equations for the $\lambda, y, g_{1}, g_{2}, g_{3}$ couplings. For input, $m_{t}=175 \mathrm{GeV}$ was used with the experimental values of the $g_{1}, g_{2}, g_{3}$ gauge couplings. The 1-loop matching of the couplings and the starting scale of the RG was chosen at $m_{Z}$.
Results from [8] exhibit the unstable Standard Model effective potential for $m_{H}=$ $52 \mathrm{GeV}$ and $m_{t}=175 \mathrm{GeV}$, where the instability appears at $\phi=1 \mathrm{TeV}$. The lower bound shown in Figure 1 is also taken from [8]. The finite width of the lower bound is an estimate of the uncertainty of the theoretical calculation, including the effect of unconsidered higher-order contributions. The strict lower bound for the Higgs mass can be further refined if one allows the ground state to be unstable, but demands that the time required to tunnel away from the local minimum at $v=$ $246 \mathrm{GeV}$ is longer than the lifetime of the universe [37, 38, 39, 40, 41].

It is clear that the current experimental limits on $m_{H}$ bring the lower bound into play. For example, a Higgs boson with a mass of $100 \mathrm{GeV}$ should indicate a breakdown of the Standard Model around $50 \mathrm{TeV}$. However, a Higgs mass in the range $160-180 \mathrm{GeV}$ apparently allows

the Standard Model to be valid all the way up to the Planck scale. The occurrence of the vacuum 
instability mostly relies on the relative magnitudes of $\lambda^{2}$ and $y^{4}$ while both renormalized couplings can remain small and all three gauge couplings of the SM are included. The perturbative RG approach, if cutoff effects can be safely ignored, seems to be on solid footing. However, cutoff effects played an important role in Top-Higgs Yukawa models where only the Higgs coupling $\lambda$ and Yukawa coupling $y$ drive the dynamics. In this approximation we have shown that vacuum instability cannot be induced with the SM Higgs potential in the cutoff Lagrangian (the possible role of higher dimensional operators to induce vacuum instability remains unclear, as we noted earlier). However, in the phenomenological application, all five couplings are running and it is important to ask: for the cutoff $\Lambda$ at or below the Planck scale $M_{P}$, should we expect Top quark induced vacuum instability with the SM cutoff Lagrangian without adding new operators? Do we expect a qualitatively different picture when compared to the Top-Higgs Yukawa model? From Figure 4 we find that the running $\lambda$ turns negative below the Planck scale for Higgs mass values lower than $135 \mathrm{GeV}$ and remains negative when $M_{P}$ is reached. Further lowering the Higgs mass lowers the scale where $\lambda$ turns negative. It remains unclear how these RG flows would be effected by holding $\lambda_{0}>0$ in the SM Higgs Lagrangian at some cutoff scale $\Lambda$. How some higher dimensional operators might provide a well-defined cutoff theory for the choice $\lambda_{0}<0$ will require further investigation.

\section{Higgs mass lower bound from the lattice}

We would like to outline and implement the first step of a robust strategy to calculate the lower Higgs mass bound as a function of the lattice momentum cutoff. The question about breaking Euclidean invariance with the lattice cutoff will eventually have to be addressed also.

\subsection{Yukawa couplings of the Top and Bottom quarks}

The third, heaviest generation of quarks consists of the left-handed $S U(2)$ top-bottom doublet $Q_{\mathrm{L}}=\left(\begin{array}{c}t_{L} \\ b_{L}\end{array}\right)$ and the corresponding right-handed $S U(2)$ singlets $t_{\mathrm{R}}, b_{\mathrm{R}}$. The complex $S U(2)$ doublet Higgs field $\Phi(x)$ with $U(1)$ hypercharge $Y=1$ is $\Phi=\left(\begin{array}{c}\phi^{+} \\ \phi^{0}\end{array}\right)$ where the suffixes,+ 0 characterize the electric charge $+1,0$ of the components. Since $\phi^{+}$and $\phi^{0}$ are complex, we can introduce four real components, $\Phi=\left(\begin{array}{c}\phi_{1}+i \phi_{2} \\ i \phi_{3}+\phi_{4}\end{array}\right)$ and the Higgs potential will have $\mathrm{O}(4)$ symmetry, with broken custodial $\mathrm{O}$ (3) symmetry, if the Yukawa couplings $y_{t}$ and $y_{b}$, defined below, are different. The Higgs potential in the complex doublet notation has the form,

$$
V(\Phi)=\frac{1}{2} m^{2} \Phi^{\dagger} \Phi+\frac{\lambda}{24}\left(\Phi^{\dagger} \Phi\right)^{2} .
$$

The Higgs field acquires a vacuum expectation value responsible for the spontaneous electroweak symmetry breaking with $\left\langle\phi_{4}\right\rangle=v$ and the first three components vanishing. The vacuum expectation value $v$ can be related to the Higgs coupling constant by $v=\sqrt{3 / \lambda} m_{H}$ with the relation between the Higgs mass $m_{H}$ and $m$ given by Equation (2.7).

Of the four Higgs components three represent Goldstone degrees of freedom, which at finite weak gauge coupling become the longitudinal degrees of freedom of the massive weak gauge bosons with mass $m_{W}=v g_{2} / 2$. The fourth component corresponds to the physical Higgs boson field. We do not use the Higgs mechanism in the limit of zero weak gauge couplings and keep all four Higgs field components where the $\phi_{1}, \phi_{2}, \phi_{3}$ fluctuations represent Goldstone particles with 
the symmetry breaking in the $\phi_{4}$ direction. In the SM Lagrangian all four Higgs components are treated on equal footing where $\mathscr{L}_{\text {Yukawa }}$ describes the interactions of the $S U(2)_{L}$ doublet Higgs field with the quark fields

$$
\mathscr{L}_{\text {Yukawa }}=y_{t} \cdot \bar{Q}_{\mathrm{L}} \Phi^{c} t_{R}+y_{b} \cdot \bar{Q}_{\mathrm{L}} \Phi b_{R}+\text { h.c. }
$$

$\Phi^{c}=i \tau_{2} \Phi^{*}$ is the charge conjugate of $\Phi, \tau_{2}$ the second Pauli matrix, $y_{t}, y_{b}$ are the top and bottom Yukawa couplings, respectively. When they are equal, the $\mathrm{O}(3)$ custodial symmetry of the Higgs potential is preserved after symmetry breaking. For unequal couplings, only the $S U(2)_{L}$ symmetry of the Lagrangian is maintained. It is easy to write out the Yukawa couplings in components:

$$
\begin{aligned}
\mathscr{L}_{\text {Yukawa }}= & y_{t}\left\{\bar{t}_{L}\left(\phi_{4}-i \phi_{3}\right) t_{R}+\bar{b}_{L}\left(i \phi_{2}-\phi_{1}\right) t_{R}\right\}+ \\
& y_{b}\left\{\bar{t}_{L}\left(\phi_{1}+i \phi_{2}\right) b_{R}+\bar{b}_{L}\left(i \phi_{3}+\phi_{4}\right) b_{R}\right\}+\text { h.c. }
\end{aligned}
$$

All masses are proportional to $v$ as they are induced by spontaneous symmetry breaking.

\subsection{One-component Top-Higgs Yukawa model}

We have used lattice simulations to study the Higgs-Yukawa model with a single real scalar field coupled to the Top quark with three colors using chiral overlap fermions. This theory has only a Higgs particle and no Goldstone bosons, and the Top quark color indices correspond to three degenerate fermions. We will not be able to calculate a lower bound directly relevant to phenomenology. Our purpose here is to explain in a simpler model how this non-perturbative calculation can be applied to a more realistic approximation of the Standard Model.

The Yukawa interaction Lagrangian in Equation (5.4) has a straightforward chiral lattice implementation in the overlap formulation where the chiral left-handed and right-handed fermion components are precisely defined. The simulation of the full doublet with the heavy Top and much lighter b quark would be very difficult on the lattice with two very different mass scales for $m_{t}$ and $m_{b}$ after spontaneous symmetry breaking.

One could choose for a pilot study the degenerate case $y_{t}=y_{b}$ which has a recent lattice implementation $[42,43]$. In this limit, there are three massless Goldstone particles contributing to Top-Higgs dynamics. When the weak gauge couplings are turned on, the massless Goldstone modes become the longitudinal components of the massive weak gauge bosons via the HiggsKibble mechanism. The limitation of the four-component model with degenerate quark doublet is the artificially enhanced fermion feedback into Higgs dynamics.

Although the degenerate model of the Top and Bottom quarks is easy to accommodate in our Higgs lattice toolbox, we chose the single component Higgs Yukawa model for our pilot study with only the Top quark included. When the weak gauge couplings are turned on, one can choose unitary gauge to eliminate the three Goldstone components. In this gauge, ignoring the weak gauge coupling effects to leading order, one is left with diagonal Top and Bottom quark Yukawa couplings where the $\mathrm{b}$ quark is decoupled in the $y_{b}=0$ limit. This is not a full justification for keeping the single Higgs field only, and the price to pay is the absence of feedback from the Goldstone modes into Higgs dynamics. Since the primary purpose of the initial phase of our Higgs project is to develop a comprehensive Higgs lattice toolbox and test its various uses, the limited one-component Higgs field dynamics will provide very useful information. The next logical step will be to restore the four components of the Higgs field which requires the b quark, and break the mass degeneracy moving toward the $y_{b} \ll y_{t}$ limit. 


\subsection{Phase diagram with chiral overlap fermions}

Lattice Yukawa models with staggered and Wilson fermions were studied before [44, 45, 46]. In this work, we adopted the overlap fermion operator to represent the chiral Yukawa coupling between the Top quark fermion field and the Higgs field. Although this is the most demanding choice for dynamical fermion simulations, staggered and Wilson fermions are not suitable for our goals. We discussed some difficulties with staggered fermions in section 3. The difficulties with Wilson fermions are worse. It turns out to be impossible to tune to the critical surface of the TopHiggs lattice Yukawa model with Wilson fermions while keeping the Wilson doublers on the cutoff scale. This is different from QCD applications of Wilson fermions.

Our massless overlap Dirac operator is defined as $a \cdot D=1+\gamma_{5} \operatorname{sign}\left(\mathrm{H}_{\mathrm{w}}\right)$ with $H_{w}=\gamma_{5} D_{w}$ where $D_{w}$ is the usual Wilson-Dirac matrix with a negative mass which for $a=1$ has the form

$$
\left(D_{w}\right)_{y x}=3 \delta_{x y}-\frac{1}{2} \sum_{\mu}\left(\left(1+\gamma_{\mu}\right) U_{\mu}(x-y) \delta_{x, y+\mu}+\left(1-\gamma_{\mu}\right) U_{\mu}^{\dagger}(x) \delta_{x, y-\mu}\right) .
$$

Using the modified $\hat{\gamma}_{5}=\gamma_{5}(1-a D)$ gamma matrix, we define two projection operators, $P_{ \pm}=$ $\frac{1}{2}\left(1 \pm \gamma_{5}\right), \widehat{P}_{ \pm}=\frac{1}{2}\left(1 \pm \hat{\gamma}_{5}\right)$, and chiral fermion components, $\bar{\psi}_{L, R}=\bar{\psi} P_{ \pm}, \psi_{R, L}=\widehat{P}_{ \pm} \psi$. The scalar and pseudoscalar densities are given by $S(x)=\bar{\psi}_{L} \psi_{R}+\bar{\psi}_{R} \psi_{L}=\bar{\psi}\left(1-\frac{a}{2} D\right) \psi$ and $P(x)=\bar{\psi}_{L} \psi_{R}-$ $\bar{\psi}_{R} \psi_{L}=\bar{\psi} \gamma_{5}\left(1-\frac{a}{2} D\right) \psi$.

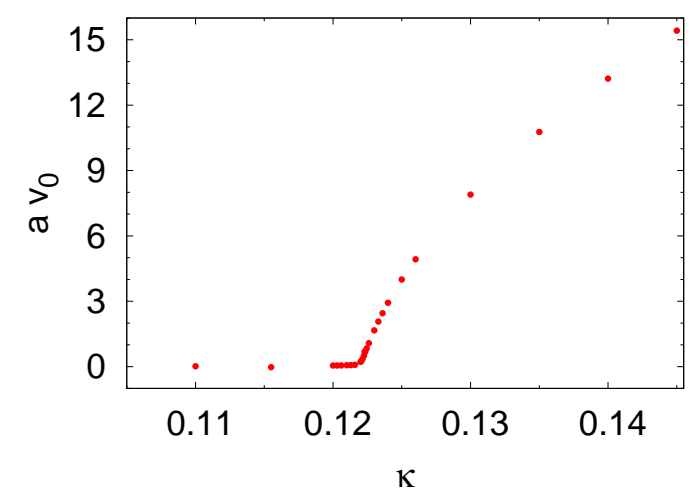

Figure 5: The vacuum expectation value of the lattice field $\phi_{0}$ is plotted in lattice spacing units $a$ as a function of the hopping parameter for fixed values of $\tilde{\lambda}_{0}=10^{-4}$, $\tilde{y}_{0}=0.35$ with 3 colors of the Top quark. The lattice size is $12^{3} \times 24$ for the plotted data. The complete phase diagram can be mapped out by varying $\tilde{\lambda}_{0}$ and $\tilde{y}_{0}$ to determine $\kappa_{c}\left(\tilde{\lambda}_{0}, \tilde{y}_{0}\right)$. non-perturbative lattice simulations. This is shown in Figure 5 where the critical critical hopping parameter for a particular choice of bare couplings is calculated. The Higgs part of the lattice Lagrangian is parametrized in the simulations as

$$
\mathscr{L}=-2 \kappa \sum_{\mu} \tilde{\phi}_{0}(x) \tilde{\phi}_{0}(x+\mu)+\tilde{\phi}_{0}^{2}(x)+\tilde{\lambda}_{0}\left(\tilde{\phi}_{0}^{2}(x)-1\right)^{2},
$$


with $\phi_{0}=\sqrt{2 \kappa} \tilde{\phi}_{0}$, and rescaled notation $\tilde{y}_{0}=y_{0} \sqrt{2 \kappa}$ for the Yukawa coupling. The odd number of colors of the single fermion required the application of the Rational Hybrid Monte Carlo (RHMC) algorithm for chiral overlap fermion. The first new code we developed was based on [47, 48]. This is the code which is mostly used in our Top-Higgs-QCD simulations. We also developed a special FFT version of the RHMC algorithm which exploited the special structure of the Yukawa coupling in the overlap Dirac operator of the Top-Higgs model. In the FFT code, Fourier acceleration is used in the evolution of the molecular dynamics trajectories which significantly reduced the autocorrelation time between independent configurations. The details of our RHMC algorithms will be described elsewhere.

\subsection{Comparison of large $\mathrm{N}_{\mathrm{F}}$ and Monte-Carlo results}

The algorithm was thoroughly tested in the large $N_{F}$ expansion of the model where we simulated a sequence of $N_{F}$ fermions, each with 3 colors, which can also be interpreted as the Top quark with $3 N_{F}$ colors. The $N_{F} \rightarrow \infty$ limit of the vacuum expectation value $v$ and the Top mass $m_{t}$ were calculated in rescaled $\lambda_{0} / N_{F}$ and $y_{0} / \sqrt{N_{F}}$ variables for the finite volumes of the simulations, for fixed value of $m_{0}^{2}$. For a particular choice of the rescaled couplings, $v$ and $m_{t}$ are plotted in Figure 6 as a function of $1 / N_{F}$. The largest number of fermions was $3 N_{F}=60$ in the
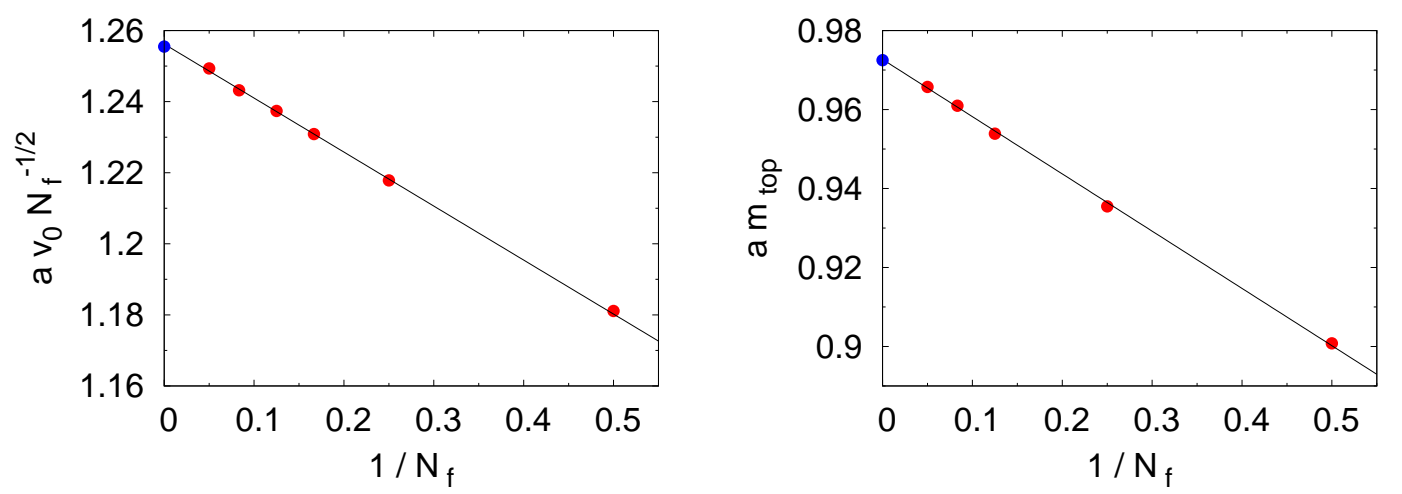

Figure 6: The vacuum expectation value $v$ of the scaled Higgs filed is plotted on the left as a function of $1 / N_{F}$ for $3 N_{F}$ fermion degrees of freedom. The blue dot marks the $1 / N_{F} \rightarrow 0$ limit. The right side plot shows the Top mass as a function of $1 / N_{F}$ with the blue dot marking the calculated $1 / N_{F} \rightarrow 0$ limit. The lattice size was $12^{3} \times 24$ for every simulation point.

sequence. The solid line indicates the scaled asymptotic value of $v$ and $m_{t}$. The finite $N_{F}$ data were numerically fitted with an added $1 / N_{F}$ correction term which allows numerical extrapolation to the $1 / N_{F} \rightarrow 0$ limit with perfect agreement. For example, in the vev test of Figure 6 the fitted curve is $1.2562(4)-0.152(2) / N_{F}$ and the large $N_{F}$ calculation gives $1.2555(7)$ asymptotically, in excellent agreement with the simulations. The sequence of simulations were done with bare parameters $y_{0} \sqrt{N_{F}}=0.7184, \lambda_{0} \cdot N_{F}=10^{-3}$, and $m_{0}^{2}=0.0637$. For the same sequence, the Top quark pole mass $m_{t}$ was fitted on the right side of Figure 6 as $0.9727(5)-0.145(2) / N_{F}$. The inverse propagator mass asymptotically is 0.9025 which converts to pole mass $m_{t}=0.9725$ at the finite lattice spacing $a$ of the simulations by the formula $a m_{t}=\ln \frac{2+a m}{2-a m}$, in perfect agreement between simulations and the large $N_{F}$ prediction. The complete agreement between the analytic large $N_{F}$ 
prediction and the Monte-Carlo results provides a very strong cross-check for the correctness of our simulation algorithm and the analytic framework.

\subsection{First results on Higgs mass lower bound}

After thorough validation of our algorithm, we turned to a preliminary determination of the Higgs mass lower bound in the single component Top-Higgs Yukawa model. The heavy Top quark will constrain the lightest possible Higgs for any given cutoff in the single component Top-Higgs Yukawa model. The starting point for simulations is the phase diagram of the theory in the bare coupling space of $m_{0}^{2}, \lambda_{0}$ and $y_{0}$. For every choice of the bare parameter set, the vacuum expectation value $v$ and the Higgs and Top masses take some values in lattice cutoff units. Keeping both the cutoff and the Top mass fixed in physical vev units, we explore all allowed bare couplings and find the lightest Higgs the theory can sustain. Repeating this procedure at various distances from the critical surface determines how the Higgs lower bound varies with the cutoff. For the Euclidean path integral to exist, we have to require $\lambda_{0} \geq 0$ in the model. We could also consider a more general Higgs action where the constraint $\lambda_{0} \geq 0$ is relaxed when positive terms like $\phi_{0}^{6}$ are added in the higher-dimensional bare coupling constant space of the bare Lagrangian. For now we do not include such terms which are part of our ongoing investigations.

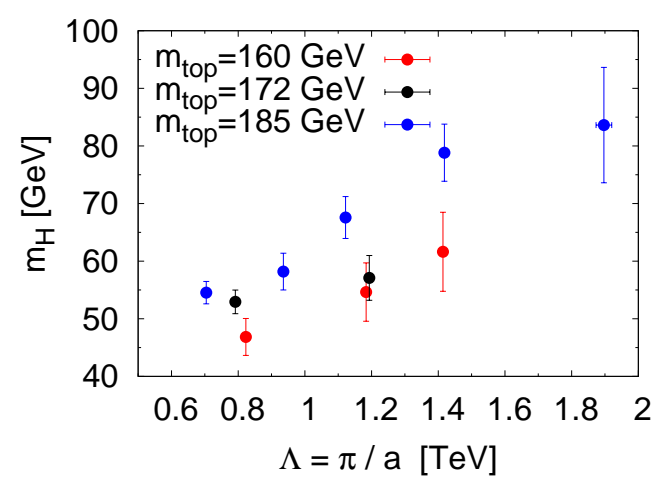

Figure 7: The lowest Higgs mass is plotted as a function of the lattice momentum cutoff for three different values of the Top mass. All simulation data are converted to physical units using $v=246 \mathrm{GeV}$.

Figure 7 displays our preliminary results which are not far from what is expected from the application of the renormalization group. Lattice artifacts will require additional interpretation in the low momentum cutoff range of the simulations.

\section{Adding the QCD gauge coupling}

Our algorithm and simulation code has been extended to the Top-Higgs-QCD code of three coupling constants. The only change is to include the SU(3) matrix link variables in the Wilson operator of Equation (5.4) in our construction of the chiral overlap operator. The numerical determination of the phase diagram and the Higgs mass lower bound in the extended model with $\lambda, y, g_{3}$ couplings (Top-Higgs-QCD model) is part of our ongoing Higgs project.

\section{Higgs mass upper bound and the heavy Higgs particle}

In this section we will review earlier results on the Higgs mass upper bound from lattice calculations and illustrate with the higher derivative (Lee-Wick) extension how a heavy particle might be exhibited without contradictions with Electroweak precision data.

\subsection{Higgs sector as an effective field theory}

In the Wilsonian view of section 4, the Standard Model is expected to have some yet unknown UV completion above a certain energy threshold $\Lambda_{0}$. This threshold could be as high as $\Lambda_{0}=$ 
$M_{\text {Planck }}$, or as low as $\Lambda_{0}=1 \mathrm{TeV}$. Below scale $\Lambda_{0}$ the SM is described by the familiar degrees of freedom for the known particles, including fermions and gauge bosons, in addition to the fourcomponent Higgs field. For illustration, we will choose $\Lambda_{0}=M_{\text {Planck }}$ first in the description of the Higgs sector without gauge and Yukawa couplings. Generalization to the full Standard Model does not add to the purpose of the discussion here. Lowering the cutoff into the $\mathrm{TeV}$ range will be part of the discussion. If the Higgs sector is treated as an effective theory, the regulator is chosen for us as an intrinsic part of the theory. Euclidean four-momenta are smoothly cut off when their lengths exceed some scale $\Lambda_{0}$. In this way all momentum integrals are made manifestly convergent, and no infinities are encountered. The simplest choice is an exponential cutoff function in the propagators,

$$
K_{\Lambda}(p)=\exp \left[-\frac{p^{2}}{\Lambda_{0}^{2}}\right]
$$

which can be built into the Lagrangian $\mathscr{L}\left(\Lambda_{0}\right)$ for non-perturbative calculations. A mass term could have been added to $p^{2}$ in Eq. (6.1) but we simplified the notation for this qualitative discussion. The general O(4) Higgs Lagrangian at scale $\Lambda_{0}=M_{\text {Planck }}$ is given by

$$
\begin{aligned}
\mathscr{L}_{\text {Higgs }} & =\frac{1}{2} \partial_{\mu} \phi^{a} \partial^{\mu} \phi^{a}+\frac{1}{2} \mu_{0}^{2} \phi^{a} \phi^{a}+\frac{\lambda_{0}}{4 !}\left(\phi^{a} \phi^{a}\right)^{2} \\
& +\frac{c_{6}}{M_{\text {Planck }}^{2}} \square \phi^{a} \square \phi^{a}+\frac{\lambda_{6}}{M_{\text {Planck }}^{2}}\left(\phi^{a} \phi^{a}\right)^{3}+\frac{c_{8}}{M_{\text {Planck }}^{4}} \square \partial_{\mu} \phi^{a} \square \partial^{\mu} \phi^{a}+\ldots,
\end{aligned}
$$

where summation is implied over $a=1,2,3,4$. Only a few higher dimensional operators are included for illustration and the exponential cutoff is implicitly understood in the functional integral built on the Lagrangian of Eq. (6.2).

\subsection{Higgs mass upper bound from the lattice}

The highest allowed Higgs mass from the Lagrangian of Eq. (6.2) was investigated before, using lattice cutoff with $c_{6}, \lambda_{6}, c_{8}$ and all other higher dimensional couplings set to zero. Corrections from the higher dimensional operators are expected to be small, of the order of powers of $m_{H} / M_{\text {Planck }}$ unless the couplings $c_{6}, \lambda_{6}, c_{8}$, or any of the other higher dimensional couplings are pushed toward asymptotically large values. It is a limit which is considered artificial and far outside naturalness bounds.

Convincing evidence for the Higgs upper bound and its numerical value comes from lattice calculations $[49,50]$ where the derivatives are replaced by finite lattice differences giving up Euclidean invariance on the Planck scale. The advantage of the lattice approach is that the full $\lambda_{0}$ range can be scanned from 0 to $\infty$. This is important if the Higgs self-interaction is a marginally (logarithmically) irrelevant operator in the triviality scenario. In the limit of infinite cutoff, the largest allowed Higgs mass would be driven to zero (triviality of the renormalized Higgs coupling), but with the cutoff at the Planck scale we will get a definitive nonvanishing upper bound which is saturated at $\lambda_{0}=\infty$ in the lattice approximation. The renormalized Higgs coupling at low energy can be defined as the ratio $\lambda_{R}=3 m_{H}^{2} / v^{2}$ where $v=246 \mathrm{GeV}$ is the vev of the Higgs field (the fourth component of the $\mathrm{O}(4)$ field), and $m_{R}$ is a renormalized Higgs propagator mass which is related in two-loop perturbation theory to the physical Higgs mass by the relation $m_{H}=m_{R}\left[1+\frac{1}{8192 \pi^{2}} \lambda_{R}^{2}\right]$. 
Based on non-perturbative lattice studies, we expect that the largest Higgs mass is obtained in the $\lambda_{0} \rightarrow \infty$ limit. For any choice of $\lambda_{0}$ in the $\mathrm{O}(\mathrm{N})$ Higgs model we have

$$
m_{R}=M_{\text {Planck }} \cdot C\left(\lambda_{0}\right) \cdot\left(\beta_{1} \lambda_{R}\right)^{-\frac{\beta_{2}}{\beta_{1}}} \exp \left(-\frac{1}{\beta_{1} \lambda_{R}}\right)\left\{1+\mathscr{O}\left(\lambda_{R}\right)\right\}
$$

with $\beta_{1}=\frac{1}{3}(N+8) \frac{1}{16 \pi^{2}}$ and $\beta_{2}=-\frac{1}{3}(3 N+14) \frac{1}{\left(16 \pi^{2}\right)^{2}}$. The relevant choice is $\mathrm{N}=4$ for the Standard Model. The non-universal amplitude $C\left(\lambda_{0}\right)$ is determined from matching to lattice calculations in the range $2 \pi \leq \Lambda / m_{H} \leq 100[49,50]$, leading to the upper bound $m_{H}=145 \mathrm{GeV}$ in the $\lambda_{0}=\infty$ limit, if the cutoff is at the Planck scale. In principle, the lattice cutoff could be replaced by the exponential cutoff function of the continuum theory. It would be required to replace the momentum square in Eq. (6.1) by its lattice version and take the inverse lattice spacing much larger than $\Lambda_{0}$. A new amplitude would emerge which could change the numerical value of the upper bound without breaking Euclidean invariance at finite cutoff. This is particularly useful when the cutoff is brought close to the low energy physical scale. In the discussion of the higher derivative extension of the Higgs sector we will show how to insert a heavy continuum cutoff scale in the theory which was turned into a practical calculation before $[11,12]$. This suggests that the insertion of the exponential cutoff scale might be feasible in practical calculations. What remains the most interesting question for LHC physics is the lowering the cutoff from the Planck scale into the TeV range. This will be illustrated next in the higher derivative extension of the Higgs sector with the scale of new physics in the $\mathrm{TeV}$ range.

\subsection{Higher derivative (Lee-Wick) Higgs sector}

An interesting extension of the Standard Model Higgs sector was proposed earlier by the addition of higher derivative operators using ideas originally discussed by Lee and Wick [11, 12, 51, 52]. Recently a complete Standard Model was constructed on similar principles [13]. Both constructions eliminate fine tuning in the Higgs sector and require ghost particles on the $\mathrm{TeV}$ scale represented by complex pole pairs in propagators with unusual physical properties. The analysis of the heavy Higgs particle from $[11,12]$ will be followed in our discussion.

In the minimal Standard Model with $S U(2)_{L} \times U(1)_{Y}$ gauge symmetry the Higgs sector is described by a complex scalar doublet $\Phi$ with quartic self-interaction as we discussed in section 5 . The Higgs potential $V\left(\Phi^{\dagger} \Phi\right)$, as defined in Equation 5.1, is $S U(2)_{L} \times U(1)_{Y}$ invariant. It also has a global $O(4) \approx S U(2)_{L} \times S U(2)_{R}$ symmetry, larger than required by the $S U(2)_{L} \times U(1)_{Y}$ gauge symmetry. Before the weak gauge couplings are switched on, it is convenient to represent the Higgs doublet with four real components $\phi^{a}$ which transform in the vector representation of $O(4)$.

We will include new higher derivative terms in the kinetic part of the $O(4)$ Higgs Lagrangian,

$$
\mathscr{L}_{H}=\frac{1}{2} \partial_{\mu} \phi^{a} \partial^{\mu} \phi^{a}-\frac{\cos (2 \Theta)}{M^{2}} \square \phi^{a} \square \phi^{a}+\frac{1}{2 M^{4}} \square \partial_{\mu} \phi^{a} \square \partial^{\mu} \phi^{a}-V\left(\phi^{a} \phi^{a}\right),
$$

where summation is implied over $a=1,2,3,4$. Also, in this subsection and the next, we use the Minkowski metric and a familiar, convenient form of the Higgs potential, $V\left(\phi^{a} \phi^{a}\right)=-\frac{1}{2} \mu^{2} \phi^{a} \phi^{a}+$ $\lambda\left(\phi^{a} \phi^{a}\right)^{2}$. The higher derivative terms of the Lagrangian in Eq. (6.4) lead to complex conjugate ghost pairs in the spectrum of the Hamilton operator. The complex conjugate pairs of energy eigenvalues of the Hamilton operator and the related complex pole pairs in the propagator of the 
scalar field ghost particles are parametrized by $\mathscr{M}=M e^{ \pm i \Theta}$. The absolute value $M$ of the complex ghost mass $\mathscr{M}$ will be set on the TeV scale. The Higgs Lagrangian $\mathscr{L}_{H}$ in Equation (6.4) describes a finite field theory without divergences, or fine tuning. It has a particularly simple form with the special choice $\Theta=\pi / 4$ of the complex ghost phase,

$$
\mathscr{L}_{H}=\frac{1}{2} \partial_{\mu} \phi^{a} \partial^{\mu} \phi^{a}+\frac{1}{2 M^{4}} \square \partial_{\mu} \phi^{a} \square \partial^{\mu} \phi^{a}-V\left(\phi^{a} \phi^{a}\right) .
$$

The $\Theta \rightarrow 0$ limit in Eq. (6.4) requires special attention. In this limit, the ghost particle becomes real and to avoid a double real pole in the propagator with problematic behavior, the choice $\Theta=0$ requires to drop the $\frac{1}{2 M^{4}} \square \partial_{\mu} \phi^{a} \square \partial^{\mu} \phi^{a}$ derivative term in the Lagrangian,

$$
\mathscr{L}_{H}=\frac{1}{2} \partial_{\mu} \phi^{a} \partial^{\mu} \phi^{a}-\frac{1}{2 M^{2}} \square \phi^{a} \square \phi^{a}-V\left(\phi^{a} \phi^{a}\right),
$$

the starting point of [13].

\subsection{Gauge and Yukawa couplings}

Gauging the Lagrangian (6.5) remained unpublished before [53]. For completeness, we present the main results. The construction of the higher derivative $\mathrm{U}(1)$ gauge Lagrangian mirrors Eq. (6.5) for the special choice $\Theta=\pi / 4$,

$$
\mathscr{L}_{\mathrm{B}}=-\frac{1}{4} F_{\mu v} F^{\mu v}-\frac{1}{4 M^{4}} \square F_{\mu v} \square F^{\mu v},
$$

with $\mathrm{U}(1)$ gauge field $B_{\mu}$ and $F_{\mu \nu}=\partial_{\mu} B_{v}-\partial_{v} B_{\mu}$. In addition to the massless gauge vector boson, the higher derivative term in Eq. (6.7) will insert a ghost particle in the spectrum of the Hamiltonian with a complex conjugate pole pair parametrized by $\mathscr{M}=M e^{ \pm i \Theta}$. For a general complex phase $\Theta$ an additional term will appear in the Lagrangian, in close analogy with the construction of Eq. (6.4).

The higher derivative Yang-Mills gauge Lagrangian for the $S U(2)_{W}$ weak gauge field $W_{\mu}$ will follow a similar construction adding the dimension eight ghost term,

$$
\mathscr{L}_{\mathrm{W}}=-\frac{1}{4} G_{\mu \nu}^{a} G^{a \mu \nu}-\frac{1}{4 M^{4}} D^{2} G_{\mu \nu}^{a} D^{2} G^{a \mu \nu},
$$

where the notation $G_{\mu \nu}^{a}=\partial_{\mu} W_{v}^{a}-\partial_{\nu} W_{\mu}^{a}+g f^{a b c} W_{\mu}^{b} W_{v}^{c}$ is used with the covariant derivative $D_{\mu}^{a b}=$ $\delta^{a b} \partial_{\mu}+g f^{a b c} W_{\mu}^{c}$. Higher derivative Lagrangians, similar to Eq. (6.8), were first introduced by Slavnov to regulate Yang-Mills theories [54].

Labeling the components of the complex $S U(2)_{L}$ Higgs-doublet field as $\Phi=\left(\begin{array}{c}\Phi^{+} \\ \Phi^{0}\end{array}\right)$ the gauged Higgs sector is described by the Lagrangian $\mathscr{L}=\mathscr{L}_{W}+\mathscr{L}_{B}+\mathscr{L}_{\text {Higgs }}$ with the Higgs Lagrangian

$$
\mathscr{L}_{\text {Higgs }}=\left(D_{\mu} \Phi\right)^{\dagger} D^{\mu} \Phi+\frac{1}{2 M^{4}}\left(D_{\mu} D^{\dagger} D \Phi\right)^{\dagger}\left(D_{\mu} D^{\dagger} D \Phi\right)-V\left(\Phi^{\dagger} \Phi\right)
$$

where the Higgs potential is $V\left(\Phi^{\dagger} \Phi\right)=-\frac{1}{2} \mu^{2} \Phi^{\dagger} \Phi+\lambda\left(\Phi^{\dagger} \Phi\right)^{2}$ and the gauge-covariant derivative is $D_{\mu} \Phi=\left(\partial_{\mu}+i \frac{g}{2} \sigma \cdot W_{\mu}+i \frac{g^{\prime}}{2} B_{\mu}\right) \Phi$. The higher derivative term in the fermion Lagrangian will take the form

$$
\mathscr{L}_{\text {fermion }}=i \bar{\Psi} \not D \Psi+\frac{i}{2 M^{4}} \bar{\Psi} \not D^{2} \not D D^{2} \Psi .
$$

Next we will briefly summarize two important features of the higher derivative Higgs sector with the ghost mass scale in the TeV range. The RG running of the Higgs coupling freezes asymptotically and a much heavier Higgs particle is allowed in extended Higgs dynamics. 


\subsection{Running Higgs coupling in the higher derivative Higgs sector}

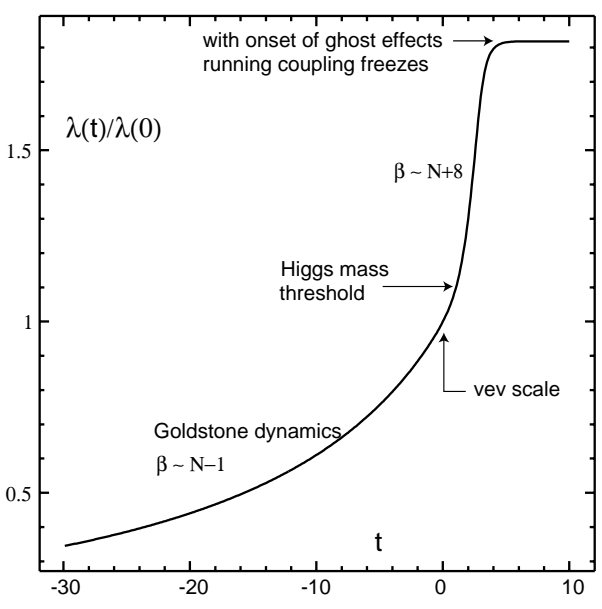

Figure 8: Running Higgs coupling in the higher Higgs sector.
This can be illustrated by calculating the scale dependent one-loop $\beta$-function within renormalized perturbation theory in the broken phase of the higher derivative $\mathrm{O}(\mathrm{N})$ Higgs sector [55, 56]. In addition to $\mathrm{N}-1$ massless Goldstone modes, there is a massive Higgs excitation and a massive complex conjugate ghost pair appears in all $\mathrm{N}$ channels, as a consequence of the new derivative term in the Lagrangian. On a low energy scale $\mu$, when $t=\log (\mu / v)$ is negative, the $\beta$-function is dominated by the Goldstone modes whose one-loop contribution is $\frac{N-1}{2 \pi^{2}} \lambda^{2}(t)$. Above the Higgs mass threshold the massive Higgs loop contribution sets in and the $\beta$-function becomes $\frac{N+8}{2 \pi^{2}} \lambda^{2}(t)$ which is the familiar one-loop form in the minimal mass independent subtraction scheme of the standard $\mathrm{O}(\mathrm{N})$ model. As $t$ in-

creases, the complex ghost loop becomes increasingly important and well beyond the ghost scale $\mathrm{M}$, for $t \gg \log (M / v e v)$, the beta-function will asymptotically vanish. The running coupling constant $\lambda(t)$ first will grow as $t$ increases, but eventually it will freeze at some asymptotic value $\lambda(\infty)$ as shown in Fig. 8. Ghost loops in the higher derivative Higgs model cancel the loops effects from the low-energy SM particles in the UV region and this 'anti-screening' effect opens up the possibility for such theories to be more strongly interacting than the standard Higgs sector.

\subsection{Scattering amplitudes}

The Higgs particle is defined as the resonance pole in the s-channel Goldstone scattering amplitude. The Goldstone amplitude can be calculated in the higher derivative Higgs sector of the $\mathrm{O}(\mathrm{N})$ Lagrangian in the large $\mathrm{N}$ approximation. In addition, the Higgs particle can be investigated directly in lattice simulations of the higher derivative model, just like in the standard Higgs sector.

In Figure 9 we plotted from $[55,57]$ the cross section as a function of the $\sqrt{s}$ center of mass energy in ghost mass units. The location of the complex Goldstone ghost pair in the scattering amplitude of the first Riemann sheet is determined by the choice of the phase angle $\Theta=\pi / 4$ in the Lagrangian of Equation (6.4). The peak in the cross section corresponds to the complex Higgs resonance pole on the second sheet of the scattering amplitude.

Also plotted in Figure 9 is the scattering phase shift as a function of $\sqrt{s}$. The phase shift has a sharp rise at the Higgs pole; however the cross section and the shape of the phase shift do not describe a standard Breit-Wigner shape in the presence of the ghosts and higher derivative Higgs dynamics. It is 'unusual' that the phase shift decreases as the energy gets through the real part of the ghost mass signaling acausal behavior in the scattering amplitude. It had been argued by Lee that this acausal behavior would only occur on microscopic scales, typical of the Compton wave length of ghosts, and it will not lead to macroscopic acausal observations. In the large $\mathrm{N}$ plots of Figure 9, the bare parameters were tuned to $m_{H}=1 \mathrm{TeV}$ for the Higgs mass with the ghost threshold 


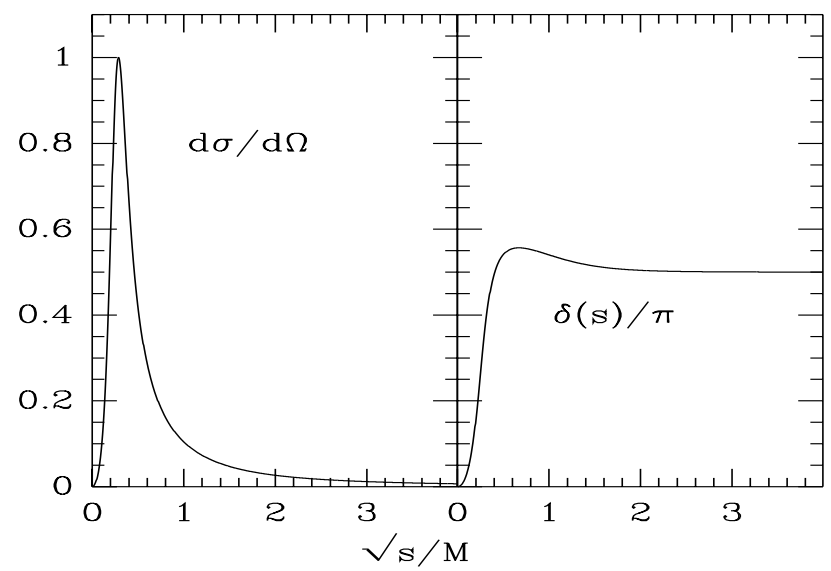

Figure 9: The Goldstone Goldstone scattering cross section and phase shift is plotted against the center of mass energy in large- $N$ expansion for the Pauli-Villars higher derivative $O(N)$ theory. The input vev value is $v=0.07$ in $M$ units. The peak corresponds to the Higgs resonance, which is at $m_{H}=0.28$ in $M$ units. The scattering cross section is completely smooth across the so-called ghost pole locations.

located at 3.6 TeV. Lattice simulations confirmed similar strongly interacting heavy Higgs physics scenarios $[55,56]$.

\subsection{Heavy Higgs particle and the $\rho$-parameter}

We discussed in the introduction that a heavy Higgs particle, beyond the $200 \mathrm{GeV}$ range, is not consistent with Electroweak precision data in the perturbative sense. Concerns were raised earlier that the heavy Higgs particle of the higher derivative Higgs sector will contribute to the Electroweak $\rho$-parameter beyond experimentally allowed limits [58]. Straightforward application

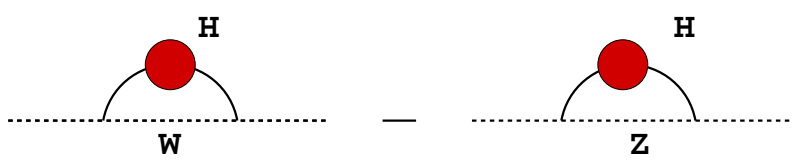

Figure 10: Higgs contribution to electroweak vacuum polarization operator.

$\rho$-parameter, of perturbative loop integrals support this concern. However, with new physics on the $\mathrm{TeV}$ scale (represented by ghost particles) the loop integrals are considerably different. A crude estimate can be made by evaluating the contribution of the vacuum polarization tensors $\Pi_{W}^{H}, \Pi_{Z}^{H}$ to the

$\rho-\left.1\right|_{\text {Higgs }}=\frac{\Pi_{W}^{H}}{M_{W, \text { tree }}^{2}}-\frac{\Pi_{Z}^{H}}{M_{Z, \text { tree }}^{2}}=-\frac{3}{4} g^{2} \int_{k^{2}<\Lambda^{2}} \frac{d^{4} k}{(2 \pi)^{4}} \frac{\Sigma_{H}\left(k^{2}\right)}{\left(k^{2}+M_{W, \text { tree }}^{2}\right)\left(k^{2}+M_{Z, \text { tree }}^{2}\right)\left(k^{2}+\Sigma_{H}\left(k^{2}\right)\right)}$,

with a sharp momentum cutoff in the $\mathrm{TeV}$ range and using the tree level Higgs self-energy operator $\Sigma_{H}\left(k^{2}\right)$. The reduction is quite large in comparison with the 1-loop perturbative formula. Replacing the cutoff integral by the Pauli-Villars regulator, which is appropriate for the higher derivative theory, we get similar reduction. The effects of the non-perturbative Higgs dynamics represented by a complicated $\Sigma_{H}\left(k^{2}\right)$ operator would have to be determined by non-perturbative simulations. If these reduction effects are not sufficient, one might need to add another Higgs doublet to the extended Higgs sector in the spirit of recent suggestions [59]. To exhibit a heavy Higgs particle as 
a broad resonance, with strong interaction and with acceptable $\rho$-parameter, remains an interesting challenge for lattice Higgs physics and model building.

\section{Acknowledgements}

J.K. is greatful for interesting discussions with J. Espinosa and D. N. would like to acknowledge helpful discussions with C. Hoelbling and K. Szabo. This research was supported by the DOE under grants DOE-FG03-97ER40546, DE-FG02-97ER25308, by the NSF under grant 0704171, by DFG under grant FO 502/1, and by the EU under grant I3HP.

\section{References}

[1] R. Barate et al. [LEP Working Group for Higgs boson searches], Phys. Lett. B 565, 61 (2003), arXiv:hep-ex/0306033.

[2] LEP Electroweak Working Group, http : / / lepewwg . web. cern.ch/LEPEWWG/.

[3] M. S. Chanowitz, Phys. Rev. D 66, 073002 (2002), arXiv : hep-ph / 0207123.

[4] M. E. Peskin and J. D. Wells, Phys. Rev. D 64, 093003 (2001), arXiv: hep-ph/ 0101342.

[5] C. Grojean, W. Skiba and J. Terning, Phys. Rev. D 73, 075008 (2006), arXiv: hep-ph / 0602154.

[6] K. Hagiwara et al. [Particle Data Group], Phys. Rev. D 66, 010001 (2002).

[7] T. Hambye and K. Riesselmann, Phys. Rev. D 55, 7255 (1997), arXiv: hep-ph / 9610272.

[8] J. A. Casas, J. R. Espinosa and M. Quiros, Phys. Lett. B 382, 374 (1996).

[9] K. Holland and J. Kuti, Nucl. Phys. Proc. Suppl. 129, 765 (2004), arXiv : hep-lat / 0308020.

[10] K. Holland, Nucl. Phys. Proc. Suppl. 140, 155 (2005), arXiv : hep-lat / 0409112.

[11] K. Jansen, J. Kuti and C. Liu, Phys. Lett. B 309, 119 (1993), arXiv: hep-lat/ 9305003.

[12] K. Jansen, J. Kuti and C. Liu, Phys. Lett. B 309, 127 (1993), arXiv: hep-lat/9305004.

[13] B. Grinstein, D. O’Connell and M. B. Wise, arXiv:0704.1845 [hep-ph] .

[14] S. R. Coleman and E. Weinberg, Phys. Rev. D 7, 1888 (1973).

[15] J. Kuti and Y. Shen, Phys. Rev. Lett. 60, 85 (1988).

[16] R. Fukuda and E. Kyriakopoulos, Nucl. Phys. B 85, 354 (1975).

[17] L. O'Raifeartaigh, A. Wipf and H. Yoneyama, Nucl. Phys. B 271, 653 (1986).

[18] L. Susskind, Phys. Rev. D 16, 3031 (1977).

[19] J. B. Kogut and L. Susskind, Phys. Rev. D 11, 395 (1975).

[20] S. Duane, A. D. Kennedy, B. J. Pendleton and D. Roweth, Phys. Lett. B 195, 216 (1987).

[21] K. G. Wilson and J. B. Kogut, Phys. Rept. 12, 75 (1974).

[22] I. V. Krive and A. D. Linde, Nucl. Phys. B 117, 265 (1976).

[23] A. D. Linde, Phys. Lett. B 70, 306 (1977).

[24] H. D. Politzer and S. Wolfram, Phys. Lett. B 82, 242 (1979) [Erratum-ibid. 83B, 421 (1979)].

[25] N. Cabibbo, L. Maiani, G. Parisi and R. Petronzio, Nucl. Phys. B 158, 295 (1979). 
[26] P. Q. Hung, Phys. Rev. Lett. 42, 873 (1979).

[27] R. A. Flores and M. Sher, Phys. Rev. D 27, 1679 (1983).

[28] M. Lindner, Z. Phys. C 31, 295 (1986).

[29] M. Sher, Phys. Rept. 179, 273 (1989).

[30] M. Lindner, M. Sher and H. W. Zaglauer, Phys. Lett. B 228, 139 (1989).

[31] C. Ford, D. R. T. Jones, P. W. Stephenson and M. B. Einhorn, Nucl. Phys. B 395, 17 (1993).

[32] M. Sher, Phys. Lett. B 317, 159 (1993) [Addendum-ibid. B 331, 448 (1994)].

[33] J. A. Casas, J. R. Espinosa and M. Quiros, Phys. Lett. B 342, 171 (1995).

[34] G. Altarelli and G. Isidori, Phys. Lett. B 337, 141 (1994).

[35] D. Boyanovsky, W. Loinaz and R. S. Willey, Phys. Rev. D 57, 100 (1998).

[36] M. B. Einhorn and D. R. T. Jones, JHEP 0704, 051 (2007), arXiv: hep-ph/ 0702295.

[37] P. B. Arnold, Phys. Rev. D 40, 613 (1989).

[38] G. W. Anderson, Phys. Lett. B 243, 265 (1990).

[39] P. Arnold and S. Vokos, Phys. Rev. D 44, 3620 (1991).

[40] J. R. Espinosa and M. Quiros, Phys. Lett. B 353, 257 (1995), arXiv: hep-ph / 9504241.

[41] G. Isidori, G. Ridolfi and A. Strumia, Nucl. Phys. B 609, 387 (2001), arXiv : hep-ph/ 0104016.

[42] P. Gerhold and K. Jansen, arXiv:0707.3849 [hep-lat ].

[43] P. Gerhold and K. Jansen, JHEP 0709, 041 (2007), arXiv: 0705.2539 [hep-lat ].

[44] L. Lin, I. Montvay, H. Wittig and G. Munster, Nucl. Phys. B 355, 511 (1991).

[45] W. Bock, A. K. De, K. Jansen, J. Jersak, T. Neuhaus and J. Smit, Nucl. Phys. B 344, 207 (1990).

[46] I. H. Lee, J. Shigemitsu and R. E. Shrock, Nucl. Phys. B 334, 265 (1990).

[47] Z. Fodor, S. D. Katz and K. K. Szabo, JHEP 0408, 003 (2004), arXiv: hep-lat/ 0311010.

[48] G. I. Egri, Z. Fodor, C. Hoelbling, S. D. Katz, D. Nogradi and K. K. Szabo, Comput. Phys. Commun. 177 (2007) 631, arXiv: hep-lat/ 0611022.

[49] J. Kuti, L. Lin and Y. Shen, Phys. Rev. Lett. 61, 678 (1988).

[50] M. Luscher and P. Weisz, Phys. Lett. B 212, 472 (1988).

[51] T. D. Lee and G. C. Wick, Phys. Rev. D 2, 1033 (1970).

[52] T. D. Lee and G. C. Wick, Nucl. Phys. B 9, 209 (1969).

[53] J. Kuti, Unpublished.

[54] T. D. Bakeyev and A. A. Slavnov, Mod. Phys. Lett. A 11, 1539 (1996), arXiv: hep-th/ 9601092 (this paper contains relevant earlier references).

[55] C. Liu, arXiv:0704.3999 [hep-ph].

[56] J. Kuti, Nucl. Phys. Proc. Suppl. 42, 113 (1995), arXiv: hep-lat/ 9502018.

[57] C. Liu, K. Jansen and J. Kuti, Nucl. Phys. Proc. Suppl. 42, 630 (1995), arXiv:hep-lat/9412034.

[58] R. S. Chivukula and E. H. Simmons, Phys. Lett. B 388, 788 (1996), arXiv: hep-ph / 9608320.

[59] R. Barbieri, L. J. Hall and V. S. Rychkov, Phys. Rev. D 74, 015007 (2006), arXiv: hep-ph/0603188. 